\title{
Aves del departamento de Cundinamarca, Colombia: conocimiento, nuevos registros y vacíos de información
}

\author{
Birds of the department of Cundinamarca, Colombia: state of knowledge, new \\ records and gaps of information
}

\section{Sergio Chaparro-Herrera, Andrea Lopera-Salazar y F. Gary Stiles}

\section{Resumen}

El departamento de Cundinamarca, Colombia, constituye un territorio con variedad de condiciones geográficas y ecosistémicas que hacen de este una de las regiones con mayor diversidad de aves en el país. Se actualizó el listado de aves y se determinaron los cambios en la composición, así como los vacíos de información. Se recopilaron los datos de campo de los autores entre 1990 y 2015, se revisó la literatura y las bases de datos y se consultaron observadores de aves expertos en la región. Fueron registradas 941 especies de las cuales 24 son endémicas, 72 casi endémicas, 1 especie extinta, 39 amenazadas a nivel nacional, 101 migratorias y 45 con migración local o altitudinal. En total, 701 especies fueron registradas por los autores en 45 localidades (48 registros nuevos para el departamento). Se concluye que existen vacíos de información en los límites departamentales, especialmente hacia la región del río Magdalena y los Llanos Orientales. Sin embargo, regiones donde existe mayor muestreo no se encuentran inventariadas en su totalidad, ya que en estas zonas se siguen encontrando nuevas especies de importancia para la ciencia.

Palabras clave. Avifauna. Cordillera Oriental. Distribución. Listado taxonómico. Registros históricos.

\begin{abstract}
The department of Cundinamarca in Colombia is an area with diverse geographic and ecosystemic characteristics, a condition that makes it one of the regions in the country with highest diversity of birds. This study updates the list of birds in the department, as well as identifies changes in composition and gaps of information, through the recopilation of field data developed by the authors from 1990 to 2015, revision of different papers and databases, and consultation of experienced birdwatchers in the region. We found 941 records of bird species, of which 24 are endemic, 72 near endemic, 1 extinct, 39 nationally threatened, 101 migratory, and 45 present local and/or altitudinal migration. Of the total number of records, 701 correspond to the authors in 45 localities (48 are new records for the department). We note that there are gaps of information in departmental boundaries, especially in the region of the Magdalena River valley and Llanos Orientales. However, regions that have been more extensively sampled are not fully inventoried since new species for science are still being found.
\end{abstract}

Keywords. Avifauna. Cordillera Oriental. Distribution. Taxonomic list. Historic records. 


\section{Introducción}

Con 1909 especies de aves que representan el $20 \%$ del total mundial y el $60 \%$ de todas las especies registradas en Suramérica, Colombia es considerado el país más diverso del planeta en este grupo taxonómico y además mantiene un flujo considerable de aves migratorias (275 especies), ya sea como migrantes recurrentes o cíclicos (Franco et al., 2009; Naranjo et al., 2012; Avendaño et al., 2017; http://www.museum.lsu. edu/ Remsen/SACCBaseline.html). De igual forma, debido a su ubicación geográfica, Colombia conjuga una variedad de componentes climáticos, hidrológicos, geológicos y geomorfológicos que conforman una amplia variedad de ecosistemas, presentando una topografía compleja dominada por los Andes (Rangel-Ch et al,. 1997; Franco y Bravo, 2005; Chaparro-Herrera et al., 2015).

Por su lado, el departamento de Cundinamarca es un territorio en el cual confluye una variedad de condiciones geográficas y ecosistémicas que van desde los páramos y el altiplano hasta el piedemonte llanero y las tierras bajas en el valle del Magdalena medio (Franco et al., 2009). Presenta 2 grandes biomas: el bosque húmedo tropical y el bosque seco, y 79 tipos de ecosistemas entre áreas naturales y transformadas siendo estas últimas las de mayor representación con un 76,80\% del departamento (Ideam et al., 2007). Este alto porcentaje de intervención se debe principalmente al manejo intensivo de la tierra, la contaminación y sobreexplotación y la aparición de numerosos centros urbanos, lo que ocasionó la extinción de una especie (Podiceps andinus, confirmada su extinción en 1982) y algunas subespecies (por ej. Anas georgica nicefori, desaparecida en la Sabana de Bogotá en 1951), la pérdida o degradación de la función ecológica de muchos ecosistemas y la reducción de bienes y servicios de calidad que estos ofrecen a las comunidades (Franco et al., 2009; Renjifo et al., 2016).

Aunque son varias las investigaciones realizadas sobre la avifauna presente en el departamento de Cundinamarca (p. ej. Munves, 1975; Lozano,
1993; Stiles, 1998; Stiles y Rosselli, 1998; ABO, 2000; López y Jiménez, 2009; Moreno y Camargo, 2008; Cortés et al., 2010; Sua y Chaparro, 2010; Gómez y Robinson, 2014; Morales-Rozo y Pachón, 2014; Chaparro-Herrera, 2015), la mayoría de estos estudios se centran en pocas localidades, principalmente en el centro del departamento o cerca de este, presentando grandes vacíos de información en sus límites.

En la actualidad, se cuenta con dos documentos en donde se recopila o sintetiza información para determinar las especies presentes en la totalidad del departamento. El primero de ellos fue realizado por Olivares (1969) y se basó principalmente en especímenes coleccionados en diferentes expediciones durante la época. En el estudio se reporta para el departamento un total de 647 especies en 94 localidades. El segundo fue realizado por Baptiste et al., en Franco et al., (2009). En esa publicación se repotan 663 especies como parte de la evaluación de especies focales para la conservación en el departamento. Dadas las dinámicas poblacionales y las fluctuaciones que se presentan a nivel espacial y temporal, así como el aumento del número de observadores de aves en el país, surgió la necesidad de realizar un listado actualizado y detallado sobre la avifauna presente en el departamento de Cundinamarca, Colombia mediante observaciones de los autores, revisión de literatura y bases de datos, entre otros. No fueron tomadas en cuenta las especies potenciales para el departamento sino solo aquellas que presentaban algún tipo de evidencia. Así mismo, con estos listados se identificaron zonas con vacíos o poca información.

Este es un trabajo realizado como complemento y homenaje al trabajo riguroso del Padre Antonio Olivares O. F. M. (1917-1975) y en pro del conocimiento y conservación de la avifauna de esta región de importancia ornitológica para el país. También es una invitación a seguir explorando, investigando y publicando registros de la avifauna del departamento de Cundinamarca. 


\section{Materiales y métodos}

Área de estudio. El departamento de Cundinamarca se encuentra ubicado en el centro de Colombia sobre la cordillera Oriental, en la región Andina; ocupa una extensión de $24210 \mathrm{~km}^{2}$ y comprende 116 municipios divididos en 15 provincias (entre los 190 y los 4375 m s.n.m.). Limita al norte con el departamento de Boyacá, al sur con los departamentos de Meta, Huila y Tolima, al occidente con el río Magdalena que lo separa de los departamentos de Tolima y Caldas y al oriente con los departamentos de Boyacá y Meta (Ramón, 1930; Olivares, 1969; http:// www.cundinamarca.gov.co/) (Figura 1).

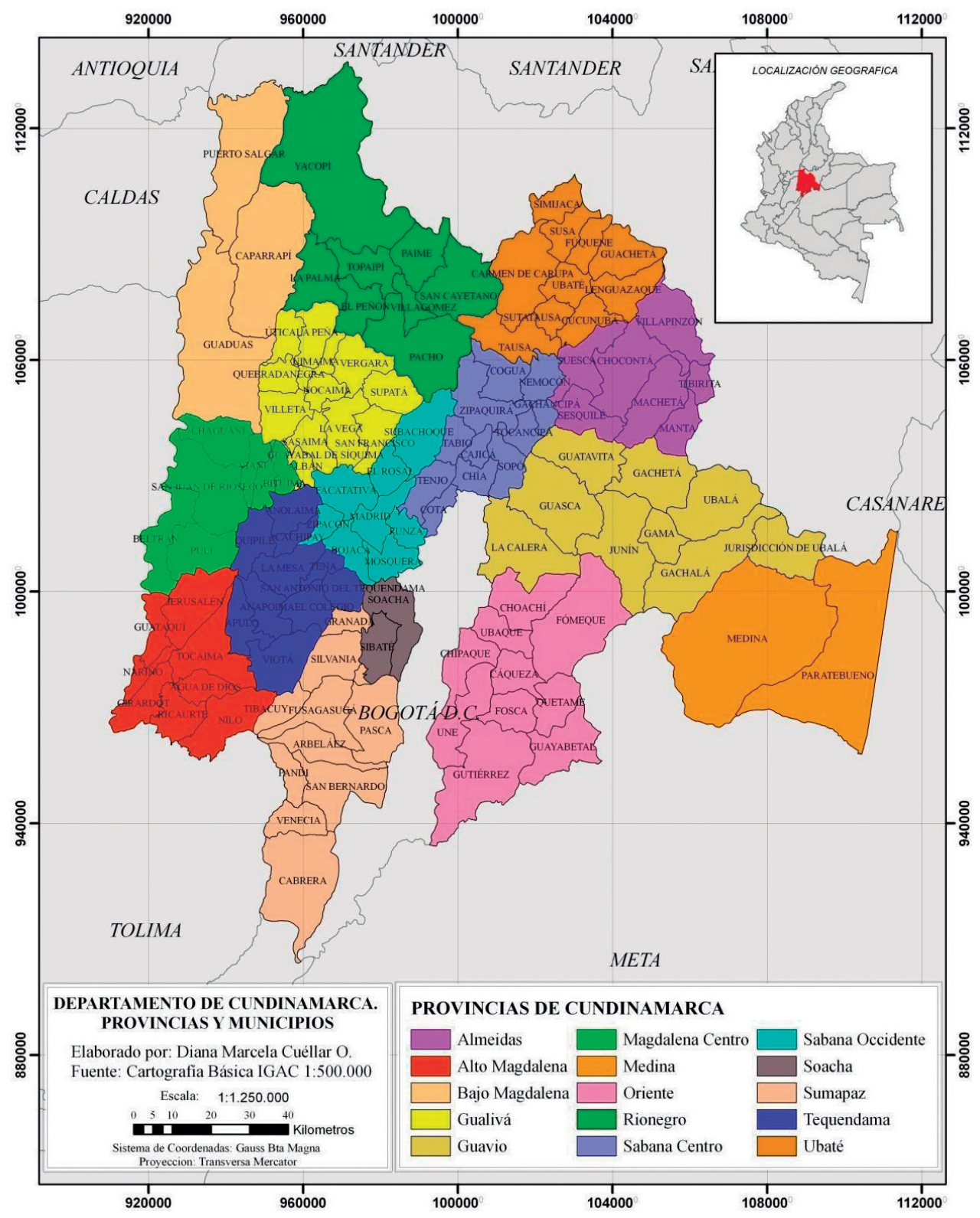

Figura 1. Ubicación, municipios y provincias del departamento de Cundinamarca. Tomado de Cuéllar (2011). 
El departamento presenta dos grandes biomas: bosque húmedo tropical y bosque seco tropical, cada uno de los cuales se encuentra subdividido en zonobiomas, orobiomas y pedobiomas (Ideam et al., 2007). En Cundinamarca el gran bioma del bosque húmedo tropical presenta los siguientes biomas: orobioma bajo de los Andes, orobioma medio de los Andes, orobioma alto de los Andes, helobiomas andinos, helobioma del Magdalena y el Caribe, helobioma de la Amazonia y Orinoquia, zonobioma húmedo tropical de la Amazonia-Orinoquia, zonobioma húmedo tropical del Magdalena-Caribe y peinobioma de la Amazonia-Orinoquia; por su lado el gran bioma del bosque seco tropical presenta el bioma: zonobioma alterno hígrico y/o subxerofítico tropical del alto Magdalena (Ideam et al., 2007) (Figura 2).

Cundinamarca, al encontrarse en la región Andina del país tiene cuatro regiones de vida (tomado de Rangel-Ch et al., 1997): páramo entre los 3300 y los 4700 m s.n.m., con diferentes tipos de vegetación como pajonales, frailejonales, matorrales, prados, chuscales, bosques achaparrados y vegetación de pantano o acuática; región Andina propiamente dicha, inicia aproximadamente a los 2400 m s.n.m. y forma una franja continua hasta los 3500-3800 m s.n.m., entre los tipos de vegetación principal se encuentran los robledales y otros tipos de bosques; región Subandina, la cual se extiende desde los 1000 hasta los 2400 m s.n.m., por las laderas de la cordillera, representado principalmente por robledales, selvas y bosques de diversos tipos. Finalmente, la región Tropical, presente desde los 100 hasta los 1100 m s.n.m. en el basamento de la cordillera con presencia de bosques ralos y matorrales principalmente (Figura 3).

El departamento tiene 79 ecosistemas, entre áreas naturales $(545,295 \mathrm{ha}, 22,72 \%$ del departamento) y transformadas (1854576 ha, 76,80 \% del departamento) (véase Ideam et al., 2007 para la clasificación, definición y extensión). Se resalta entre las áreas naturales, por ser las más extensas: herbazales del orobioma alto de los Andespáramo y entre las áreas transformadas los pastos del orobioma bajo de los Andes (Tabla 1, Figura 4).

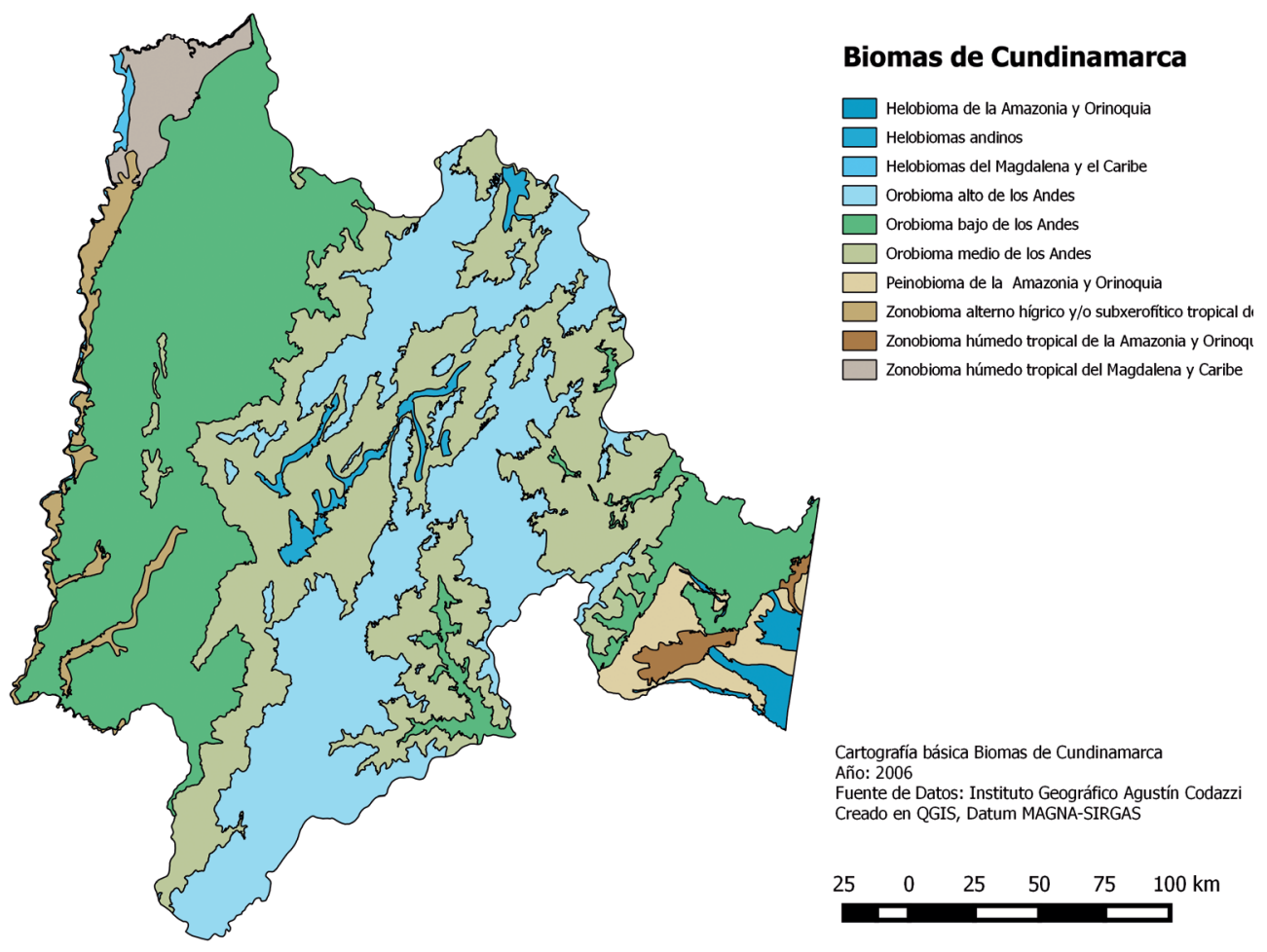

Figura 2. Mapa de los biomas presentes en el departamento de Cundinamarca. 

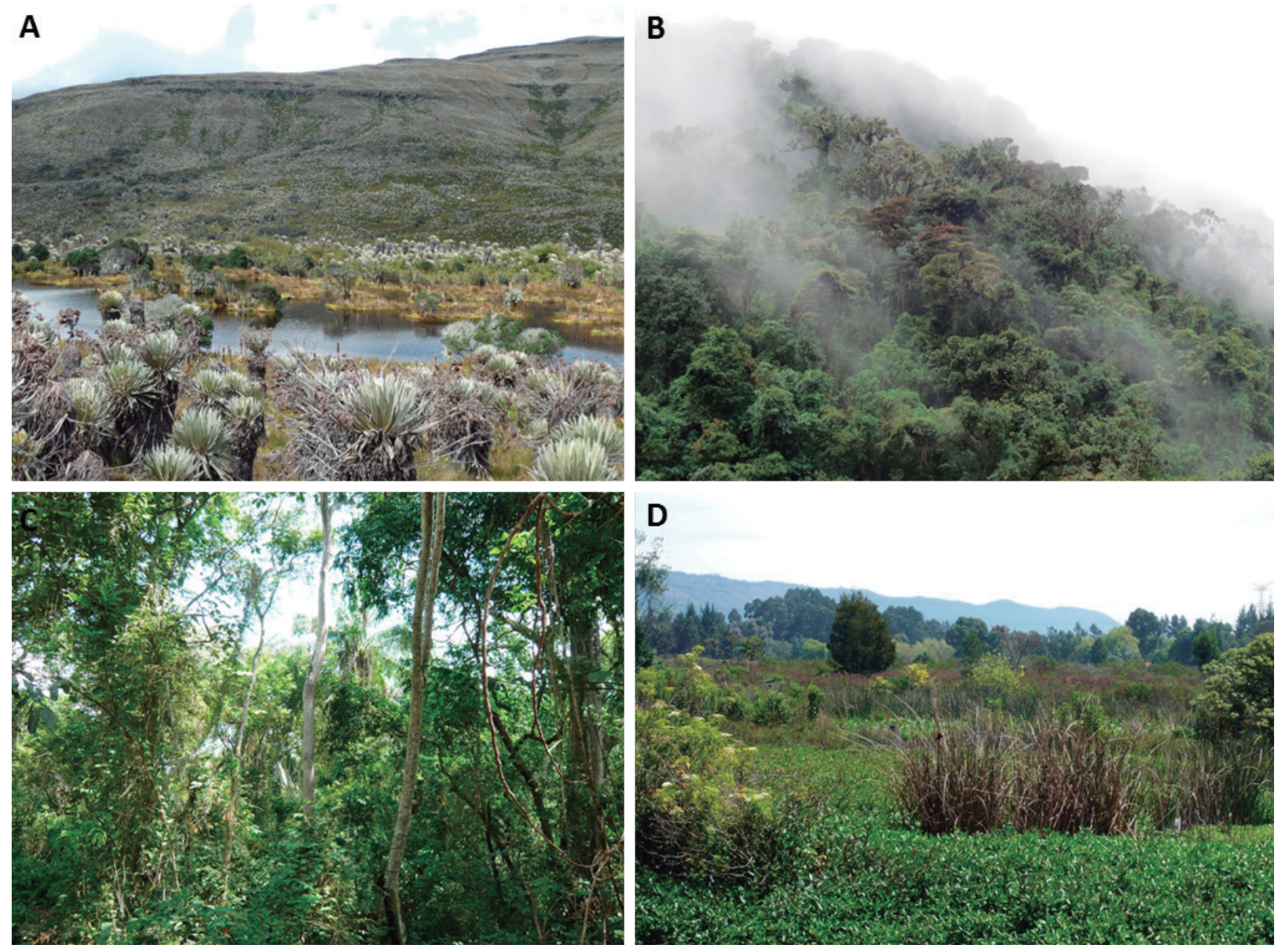

Figura 3. Algunos de los hábitats presentes en el departamento de Cundinamarca. A) Páramo, B) bosque altoandino, C) bosque seco y D) humedales. Fotografías: Sergio Chaparo-Herrera.

Tabla 1. Ecosistemas naturales y transformados destacados por su extensión en el departamento de Cundinamarca. Tomado de: Ideam et al. (2007).

\begin{tabular}{llcc}
\hline \multicolumn{1}{c}{ Ecosistema } & Área (ha) & $\begin{array}{c}\text { Representación } \\
\text { del departamento } \\
\text { (\%) }\end{array}$ \\
\hline \multirow{5}{*}{ Natural } & $\begin{array}{l}\text { Herbazales del orobioma alto de los } \\
\text { Andes-páramo }\end{array}$ & 151891 & 6,33 \\
\cline { 2 - 4 } & $\begin{array}{l}\text { Bosques naturales del orobioma } \\
\text { medio de los Andes }\end{array}$ & 107790 & 4,49 \\
\hline $\begin{array}{l}\text { Bosques naturales del orobioma alto } \\
\text { de los Andes }\end{array}$ & 84412 & 3,52 \\
\hline $\begin{array}{l}\text { Bosques naturales del orobioma bajo } \\
\text { de los Andes }\end{array}$ & 69698 & 2,90 \\
\hline $\begin{array}{l}\text { Arbustales del orobioma alto de los } \\
\text { Andes-páramo }\end{array}$ & 58192 & 2,42 \\
\hline
\end{tabular}


Cont. Tabla 1. Ecosistemas naturales y transformados destacados por su extensión en el departamento de Cundinamarca. Tomado de: Ideam et al. (2007).

\begin{tabular}{|c|c|c|c|}
\hline & Ecosistema & Área (ha) & $\begin{array}{c}\text { Representación } \\
\text { del departamento } \\
(\%)\end{array}$ \\
\hline \multirow{6}{*}{ Transfomado } & Pastos del orobioma bajo de los Andes & 355156 & 14,8 \\
\hline & $\begin{array}{l}\text { Áreas agrícolas heterogéneas del } \\
\text { orobioma bajo de los Andes }\end{array}$ & 235308 & 9,81 \\
\hline & $\begin{array}{l}\text { Áreas agrícolas heterogéneas del } \\
\text { orobioma medio de los Andes }\end{array}$ & 197333 & 8,22 \\
\hline & $\begin{array}{l}\text { Pastos del orobioma medio de los } \\
\text { Andes }\end{array}$ & 157439 & 6,56 \\
\hline & $\begin{array}{l}\text { Cultivos anuales o transitorios del } \\
\text { orobioma medio de los Andes }\end{array}$ & 138842 & 5,79 \\
\hline & Pastos del orobioma alto de los Andes & 124828 & 5,20 \\
\hline
\end{tabular}

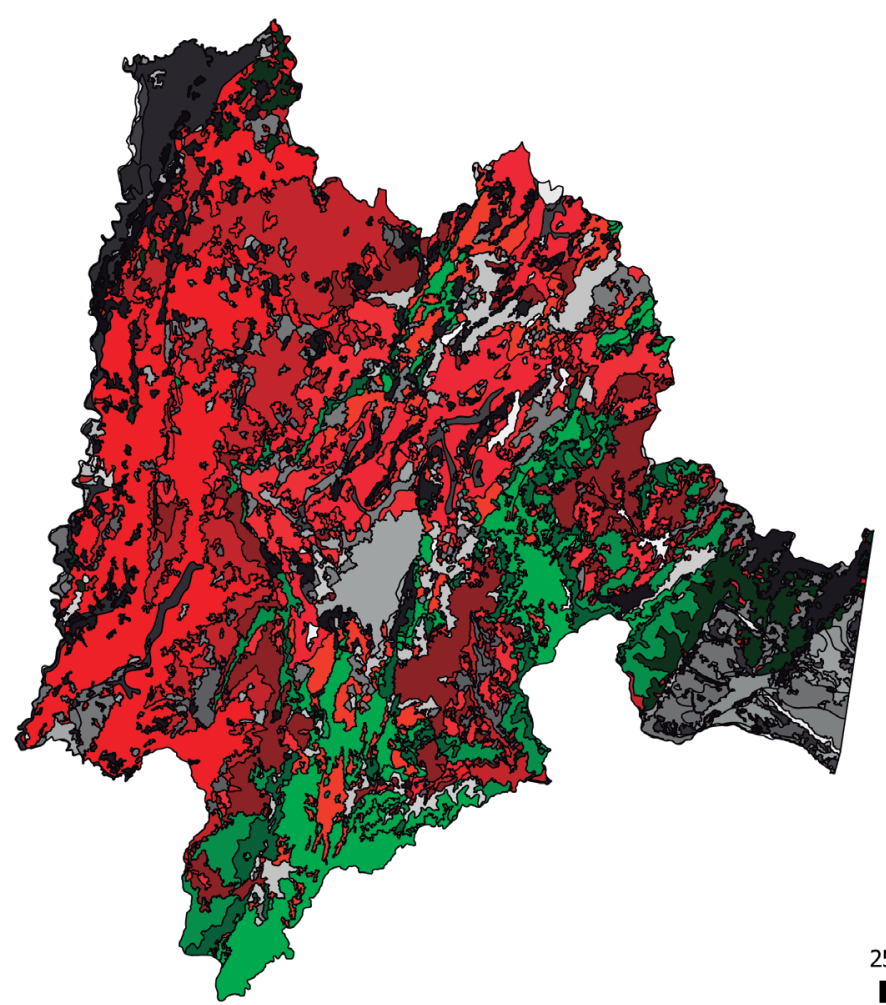

Ecosistemas predominantes en Cundinamarca

Arbustales del orobioma alto de los Andes

Bosques naturales del orobioma alto de los Andes

Bosques naturales del orobioma bajo de los Andes

Bosques naturales del orobioma medio de los Andes

Herbazales del orobioma alto de los Andes

Áreas agrícolas heterogéneas del orobioma bajo de los Andes

Áreas agrícolas heterogéneas del orobioma medio de los Andes

Cultivos anuales o transitorios del orobioma medio de los Andes

Pastos del orobioma alto de los Andes

Pastos del orobioma bajo de los Andes

Pastos del orobioma medio de los Andes

Año:2006

Fuente de datos: Instituto Geográfico Agustín Codazzi

Creado en QGIS, Datum MAGNA-SIRGAS.
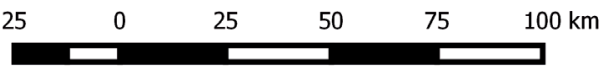

Figura 4. Mapa de los ecosistemas predominantes en Cundinamarca. 
Finalmente, el departamento cuenta con 183 áreas naturales protegidas en 10 categorías de manejo, divididas de la siguiente manera (Vásquez y Serrano, 2009): 57 Reservas de la Sociedad Civil, 47 Áreas Forestales Distritales, 44 Reservas Forestales Protectoras, 18 Parques Ecológicos Distritales, 9 Distritos de Manejo Integrado, 7 Reservas Hídricas, 5 Reservas Forestales Protectoras Productoras, 3 Santuarios Distritales de Fauna y Flora, 2 Parques Nacionales Naturales (PNN Chingaza, PNN Sumapaz) y un Área de Manejo Especial (Sierra Morena).

Se resalta por su extensión y categoría de manejo el Parque Nacional Natural Chingaza con una extensión de 76600 ha que abarca un rango altitudinal que va entre desde los 800 y hasta los 4020 m s.n.m. El 74,7 \% del parque está en Cundinamarca $(57,250$ ha) y equivale al 2,36 \% del departamento. El PNN Sumapaz tiene un área de 223,179 ha con un rango altitudinal que va desde los 700 y hasta los 4375 m s.n.m., con 132657 ha en Cundinamarca (59,4 \% del parque) que equivale al 5,47 \% del departamento (Vásquez y Serrano, 2009; Morales et al., 2007; http:/ / www. parquesnacionales.gov.co/). Estas dos áreas protegidas abarcan el 7,83 \% del departamento, siendo las áreas de conservación de biodiversidad más importantes en Cundinamarca. Sumado a estas se encuentran nueve Áreas Importantes para la Conservación de las Aves (AICA) que a pesar de no estar bajo alguna categoría de área protegida en la legislación del país (a excepción de las áreas ya legalmente constituidas), representan lugares críticamente importantes a nivel mundial para las aves y la biodiversidad en general (http://datazone.birdlife.org/country/ colombia/ibas): Serranía de las Quinchas (distribuida entre los departamentos de Boyacá, Santander y Cundinamarca-pequeña proporción en el municipio de Yacopí), Complejo lacustre de Fúquene, Cucunubá y Palacio (distribuido en los departamentos de Boyacá y Cundinamarca), Gravilleras del valle del río Siecha (localizada en los municipios de Guasca y Guatavita con un área de 1500 ha), Parque Nacional Natural Chingaza (localizado entre los departamentos de
Cundinamarca y Meta), Parque Nacional Natural Sumapaz (localizado entre los departamentos de Meta, Huila y Cundinamarca), humedales de la Sabana de Bogotá (ubicados principalmente en la ciudad de Bogotá y sus alrededores con un área de 18000 ha), bosque de Tolemaida, Piscilago y alrededores (localizado en el municipio de Nilo con 20000 ha), cerros occidentales de Tabio y Tenjo (ubicados en los municipios con su mismo nombre con un área de 411 ha) y bosques de la falla del Tequendama (ubicados sobre la vertiente occidental de la cordillera Oriental con un área de 11000 ha).

Por otra parte, los bosques de niebla del flanco occidental de la cordillera han sufrido considerables niveles de transformación, contrario a lo que ocurre en la vertiente oriental donde los bosques de niebla son los que presentan mejor estado de conservación con una extensión considerable. Sin embargo ambas vertientes de la cordillera Oriental se encuentran en un estado de fragmentación por lo que debe ser monitoreado, principalmente en la vertiente occidental (Morales y Armenteras, 2013).

Recopilación de datos. El listado se elaboró a partir de varias fuentes de información (salidas de campo, bases de datos y literatura):i) se recopilaron datos de campo de los autores (observaciones sistemáticas, observaciones libres, capturas con redes de niebla, colecta de especímenes y grabaciones vocales) en diversas localidades del departamento entre 1990 y octubre de 2015 (Anexo 1). ii) Se consultaron las bases de datos de los especímenes depositados en el Instituto de Ciencia Naturales de la Universidad Nacional (ICN, 2015) y del Instituto de Investigación de Recursos Biológicos Alexander von HumboldtIAvH (http://i2d.humboldt.org.co/ceiba/ resource.do?r=aves_iavh), la base de datos de la alianza Biomap (http://www.biomap.net) de especímenes presentes en museos de Colombia y fuera del país, la Colección de Sonidos Ambientales del Instituto Humboldt (http:/ /ipt.biodiversidad. $\mathrm{co} /$ iavh/resource? $\mathrm{r}=$ bancosonidos), la base de cantos Xenocanto Foundation (www.xeno-canto.org), 
la base de datos del Censo Nacional de Aves en Colombia (RNOA, http://ipt.sibcolombia.net/ rnoa/resource.do?r=censo_aves_rnoa), los datos consignados en el Sistema de Información de la Biodiversidad (SIB, http://ipt.sibcolombia.net/ rnoa/ resource.do?r=asocolflores, http:/ / doi. org/10.15472/qvuuko, http://ipt.biodiversidad. co/iavh / resource? r=rrbb_chingaza faunaflora_2015) y la base de datos eBird (http:/ / www.ebird.org). iii) Se revisaron artículos científicos, libros, guías de campo y literatura gris (tesis e informes técnicos) (Anexo 2). También se consultaron directamente varios observadores de aves, de manera informal, por registros nuevos para el departamento. Los registros dudosos o con falta de evidencia no fueron tenidos en cuenta en este listado. Unos pocos registros, de la base de datos eBird (http://www.ebird.org), y los cuales se encuentran en el listado final con un signo de interrogación, fueron tenidos en cuenta tras una evaluación (observador, evidencia, registros cercanos, entre otros) por parte de los autores de su posible presencia. Tampoco fueron tenidas en cuenta las especies cuya distribución potencial pudiera incluirlas en el departamento, debido a que solo se quiere presentar las especies con alguna evidencia de su presencia en este.

Para complementar la información de las especies fueron empleadas las siguientes fuentes: Endémicas (E), Casi-endémicas (CE), De Interés (DI) e Información Insuficiente (II) (ChaparroHerrera et al., 2013), Categoría de amenaza global y nacional (Renjifo et al., 2014, 2016; Ministerio de Ambiente y Desarrollo Sostenible, 2017; BirdLife International, 2017; IUCN: http:// www.iucnredlist.org/) y tipo de migración, ya sea transfonteriza, local o altitudinal (Fierro, 2009; Naranjo et al., 2012). La taxonomía sigue a SACC 2017 (julio) (http://www.museum.lsu. edu/ Remsen/SACCBaseline.html). Finalmente con las localidades visitadas por los autores, localidades mencionadas por Olivares 1969 y fuentes de información como artículos científicos, libros y literatura gris (no se tuvieron en cuenta listados aislados o con poca representatividad de muestreo), fue realizado un mapa de calor usando la estimación de densidad de Kernel en el programa Quantum GIS Geographic Information System (2017) versión 2.18.7 y el cual representa la densidad basado en el número de puntos en una ubicación, con el fin de poder determinar los vacíos de información o las localidades con menor esfuerzo de muestreo a lo largo del departamento.

\section{Resultados}

Se obtuvo un listado para el departamento de Cundinamarca de 941 especies (24 órdenes, 71 familias, 795 géneros), con 2 especies en 1 categoría taxonómica incierta (Incertae sedis, Piprites chloris y Rhodinocichla rosea). El listado del departamento representa el 49,2 \% de las 1909 especies presentes en Colombia (Avendaño et al., 2017). De estas especies 24 son endémicas, 72 casi endémicas, incluyendo en estas últimas al loro orejiamarillo (Ognorhynchus icterotis) cuya distribución histórica incluía el noroccidente de Ecuador, sin embargo, no hay certeza que dicha población se encuentre extinta en la actualidad (Devenish et al., 2009; Renjifo et al., 2014) (Figura 5). Se registran a su vez 3 especies de interés para Colombia (especie que presentan entre el 40-49\% de su área de distribución en Colombia), 1 especie con información insuficiente (Setopagis heterura) y 2 introducidas (IN) (Columba livia y Lonchura malacca); una especie extinta (Podiceps andinus), 39 nacionalmente amenazadas de las cuales 5 están en Peligro Crítico (CR), 15 En Peligro (EN), 13 Vulnerable (VU) y 5 Casi Amenazadas (NT), además de Gallinago imperialis, Falco deiroleucus y Cacicus uropygialis que presentan datos insuficientes (DD); 37 especies se encuentran en alguna categoría de amenaza global, 12 de ellas no se consideran nacionalmente amenazadas y 8 especies difieren en la categoría nacional y global, siendo de mayor grado la amenaza nacional a excepción de Leptotila conoveri considerada Vulnerable (VU) a nivel nacional y En Peligro (EN) a nivel global; de estas, 9 se encuentran En Peligro (EN), 12 Vulnerables (VU) y 15 Casi Amenazadas (NT). 
Se registraron 91 migratorias boreales (MB) y 10 migratorias australes (MA) y 1 especie errante (Anas crecca). Se resalta a Myiodynastes maculatus que tiene poblaciones migrantes del hemisferio norte y poblaciones migrantes del hemisferio sur; Setophaga cerulea, migratoria boreal en estado Vulnerable de amenaza global y nacional y Contopus cooperi, migratoria boreal Casi Amenazado global y nacionalmente, además de 3 migratorias boreales casi amenazadas a nivel global; 19 de las especies invernantes poseen poblaciones reproductivas permanentes y posiblemente se suman a estas Charadrius vociferus y Tringa melanoleuca, las cuales pueden tener eventos reproductivos en el país (Sabana de Bogotá, páramo de Sumapaz, respectivamente) (S. Chaparro-Herrera obs. per.). Adicionalmente, 44 especies presentan otro tipo de migración, bien sea local o altitudinal, de estas 3 especies se encuen-tran en categoría de amenaza Vulnerable (Anexo 3).

Las familias más representativas fueron Tyrannidae con 114 especies, seguida de Thraupidae con 98, Trochilidae con 83 y Furnariidae con 56. Del total de las especies reportadas, 701 fueron registradas por los autores en 45 localidades $(74,4 \%)$ (48 registros nuevos para el departamento principalmente en los municipios de Yacopí y UbaláMámbita) (Figura 6, Anexo 3, 4), 763 en diversas fuentes de información (81 \%) y 830 en bases de datos $(88,2 \%)$, de las cuales 738 presentan especímenes en colecciones científicas (78,4\%) (Anexo $1,2,3)$. A su vez 13 registros son nuevos para el departamento (otros autores): Anas bahamensis, observada en el municipio de Mosquera (D. Rodríguez en prensa) y Anapoima (V. Vergara com. pers.), Calidris fuscicollis, registrada en la laguna de Chisacá en el PNN Sumapaz (A. Pinto datos no publicados), Leptodon cayanensis, observada en Mámbita-Ubalá (N. León com. pers.), Morphnarchus princeps, observada en Guayabetal (A. Cuervo com. pers.), Buteo albigula, observada en el PNN Chingaza (F. Gómez com. pers.) y Bogotá (J. Velásquez com. pers.), Megascops ingens, observada en Bojacá (A. Bautista com. pers.; grabada por M. Álvarez en Medina, requiere confirmación), Nyctibius maculosus, observada en Chingaza (A. Bautista datos no publicados), Ara militaris, observado un grupo de nueve individuos en Nilo (http:/ / www.ebird.org), Furnarius leucopus, registrada en Beltrán (S. Lleras com. pers.), Chloropipo flavicapilla, observada en la laguna de Pedro Palo-Tena (D. Calderón datos no publicados), Piprites chloris, grabada vocalmente en el municipio de Medina (O. Laverde com. pers.), Setophaga caerulescens, observada en el cerro de Quininí (http://www.ebird.org, A. Caguazango com. pers.) y Tiaris fuliginosus, capturada en Nilo (A. M. González datos no publicados).

Olivares (1969) reporta para el departamento 646 especies, de las cuales 8 no presentan otros registros publicados a la fecha, en paréntesis la localidad de registro: Tinamus tao (Guaicaramo), Anas crecca (Fúquene), Gallinago imperialis (Choachí?, Siecha?), Rynchops niger (Girardot), Polytmus guainumbi (Medina), Discosura conversii (indicios colectada en Yacopí), Psittacara leucophthalmus (Guaicaramo), Phyllomyias burmeisteri (Puerto Salgar) y Laniocera hypopyrra (Guaicaramo), a su vez reporta a Chlorostilbon russatus en la laguna de Fúquene. Sin embargo, de estas especies reportadas por Olivares (1969), Tinamus tao fue registrada recientemente en Medina (O. Laverde com. pers.), R. niger observada esporádicamente en los últimos años en el Parque Metropolitano Simón Bolívar en Bogotá (S. Chaparro-Herrera obs. pers.), y en el municipio de Beltrán (S. Lleras com. pers.) y Anapoima (V. Vergara com. pers.), Psittacara leucophthalmus registrada recientemente en Medina (O. Laverde com. pers.) y P. burmeisteri fue capturado en 2016 en cercanías de Nilo (http://www.ebird.org).

De acuerdo con los registros de las colecciones biológicas consultados en Biomap (http://www. biomap.net), Instituto Humboldt: http://i2d. humboldt.org.co/ceiba/resource.do? $r=a v e s \_i a v h$ e ICN (2015), existen especímenes de 738 especies $(78,4 \%)$ de las cuales 29 son la única evidencia en el departamento, principalmente en los municipios de Medina, Paratebueno y Puerto Salgar, límites del departamento hacia oriente (Llanos Orientales) y occidente (valle del río Magdalena) (Tabla 2). 

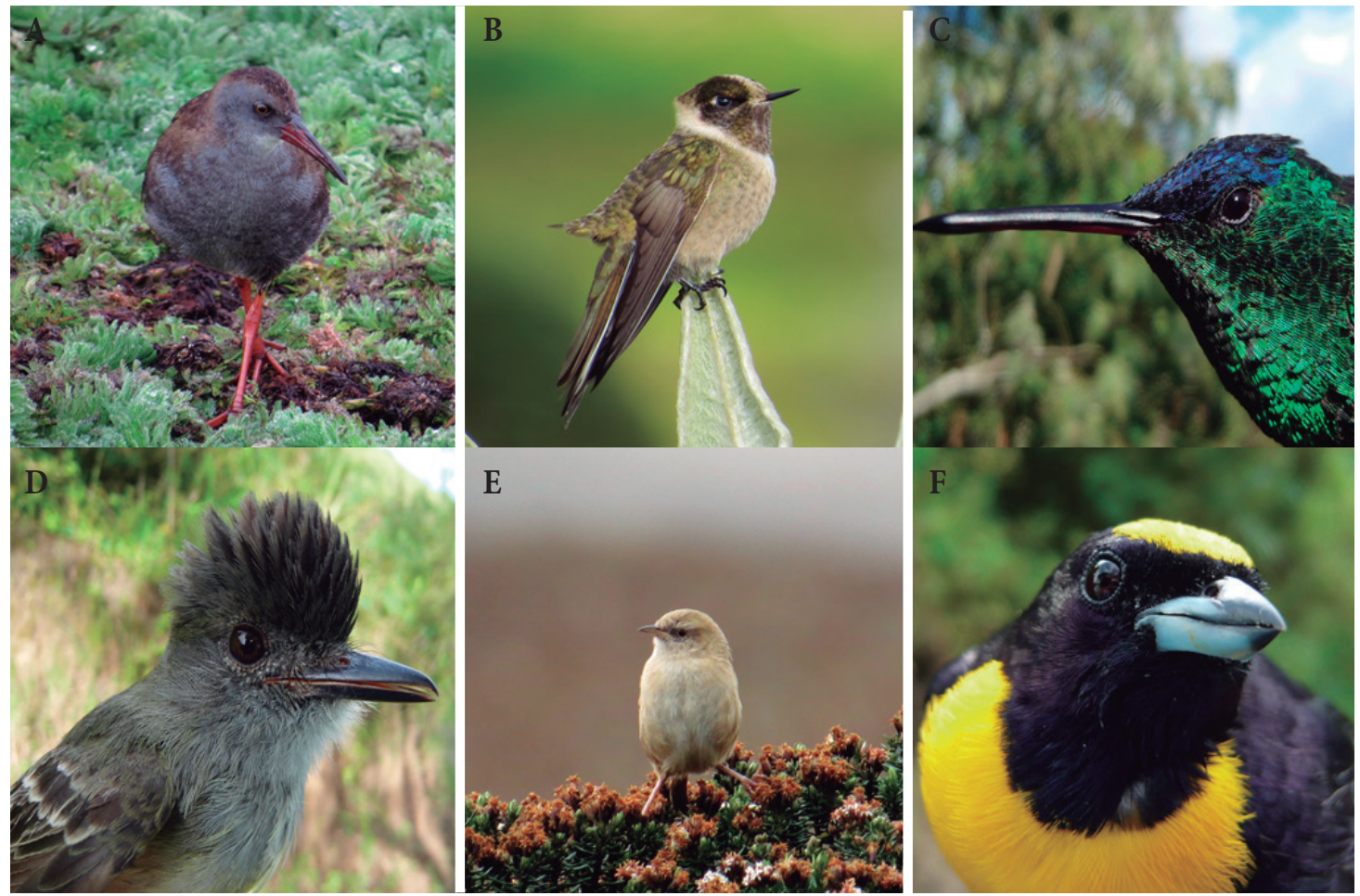

Figura 5. Algunas de las especies endémicas presentes en el departamento de Cundinamarca. A) Rallus semiplumbeus, B) Oxypogon guerinii (hembra), C) Amazilia cyanifrons, D) Myiarchus apicalis, E) Cistothorus apolinari y F) Euphonia concinna (macho). Fotografías: Sergio ChaparroHerrera.
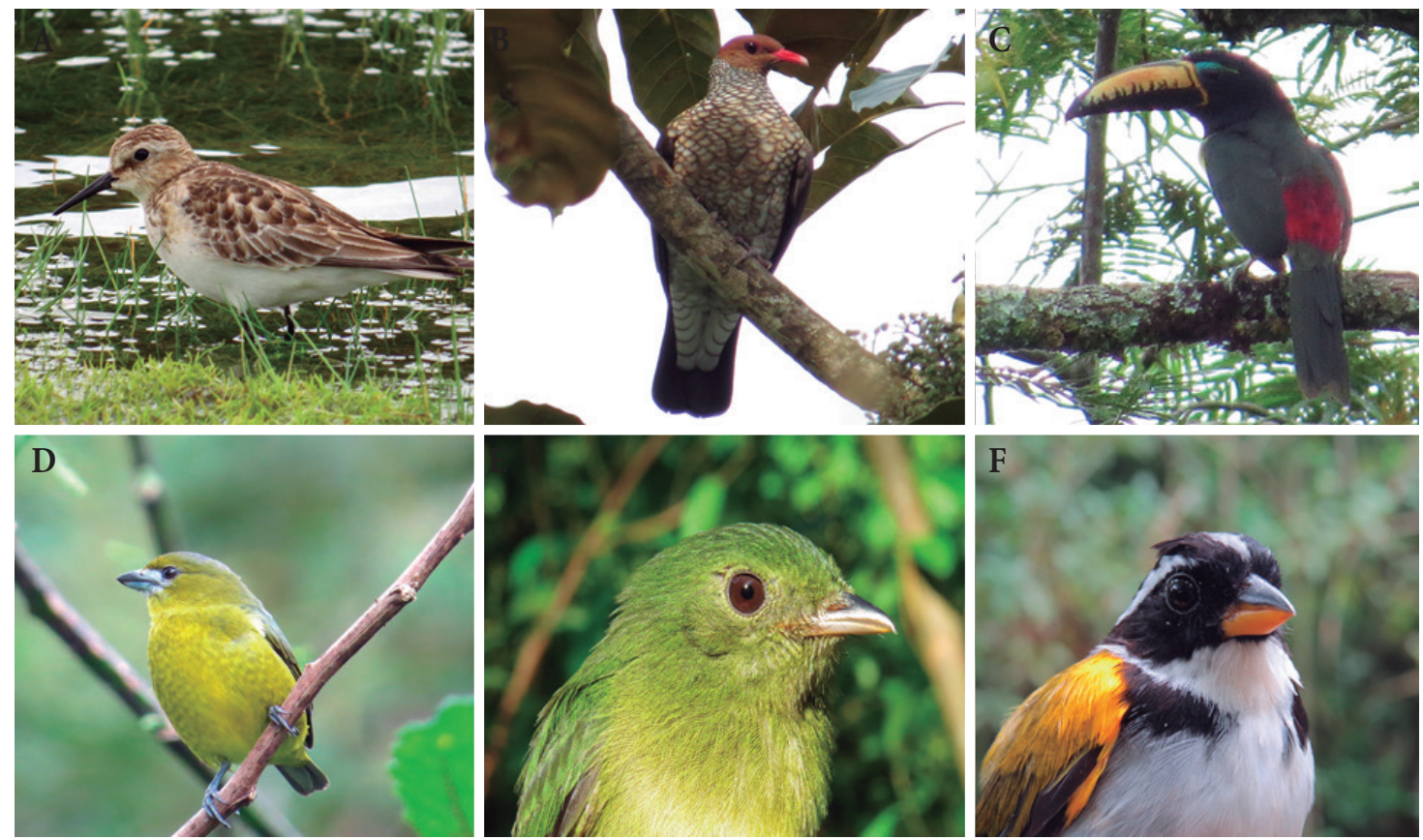

Figura 6. Alguna de las especies registradas por los autores como nuevos registros para el departamento de Cundinamarca. A) Calidris bairdii, B) Patagioenas speciosa, C) Pteroglossus inscriptus, D) Euphonia chrysopasta, E) Cryptopipo holochlora y F) Arremon taciturnus. Fotografías: Sergio Chaparro-Herrera. 
A su vez solo se conocían especímenes para Tangara mexicana en Medina (T. H. Wheeler, sin fecha) y Tangara chilensis (Mámbita-Ubalá, 1917 y Medina T. H. Wheeler, sin fecha), sin embargo en expediciones realizadas a la región por los autores en octubre de 2015 a Mámbita, municipio de Ubalá estas fueron registradas.

En cuanto a los registros sonoros consultados (Instituto Humboldt: http://ipt.biodiversidad. co/iavh/resource? $\mathrm{r}=$ bancosonidos, Xenocanto Foundation: http://www.xeno-canto.org/) se tienen grabaciones de 352 especies $(37,4 \%)$ de las cuales para 5 especies es la única evidencia de su presencia en el departamento (Tabla 2).

A su vez 49 especies presentan únicamente registros históricos, ya sea por especímenes coleccionados o por datos en Olivares (1969) (Anexo 5). Desde este punto resaltamos a 5 especies cuyos registros eran históricos, pero que en la actualidad cuenta con datos de observación, este es el caso del tinamú gris (Tinamus tao) una especie mencionada por Olivares (1969) en el municipio de Paratebueno (Guaicaramo) y registrada recientemente en Medina (O. Laverde com. pers.), el musguero de anteojos (Siptornis striaticollis) una especie bastante rara y local en todo su rango de distribución y cuyos registros son históricos (Fusagasugá 1917), pero actualmente ha sido observado en varias ocasiones en el municipio de San Francisco (M. Hernández com. pers.), la perdiz colorada (Odontophorus hyperythrus) cuyos registros se remontan a 1913 en Subia y registrada en 2006 por O. Laverde en Los Robles (Fusagasugá) (http://ipt.biodiversidad. $\mathrm{co} /$ iavh/resource? $\mathrm{r}=$ bancosonidos), el perico ojiblanco (Psittacara leucophthalmus) registrada en 1946 en Paratebueno (Guaicaramo, río Guavio), Cundinamarca-Meta y registrada recientemente en Medina (O. Laverde com. pers.) y el hormiguero dorsicastaño (Poliocrania exsul) registrada en Puerto Salgar en 1968 y observada en 2016 en Beltrán (S. Lleras com. pers.) En el listado no es tenido en cuenta al heliangelus de Bogotá (Heliangelus zusii), un espécimen de origen y distribución desconocida y proveniente de las denominadas "pieles de
Bogotá" (1909) con posible origen en la cordillera Oriental cerca de Bogotá o de la cordillera Central (Graves, 1993; Renjifo et al., 2002) y cuyos estudios moleculares recientes sugieren que no es una especie válida sino el resultado de la hibridación entre una hembra Aglaiocercus kingii y un macho de otro colibrí (Pérez-Emán et al., 2017).

Otros registros de importancia al ser los únicos datos de la presencia de algunas especies en el departamento son (tomado de http:// www.ebird.org, algunos de ellos requieren confirmación, representados con un signo de interrogación): Tinamus major? (Reserva Mana Dulce entre Agua de Dios-Nilo, y laguna de Pedro Palo-Tena), Eudocimus albus? (Puerto Salgar), Theristicus caudatus (Anapoima), Platalea ajaja (Reserva Mana Dulce entre Agua de DiosNilo), Cathartes burrovianus (arriba de la Dorada), Geranospiza caerulescens (arriba de la Dorada, Chinauta y Apulo), Chaetura cinereiventris (Tabacal-La Vega, La Aguadita-Fusagasugá, parque La Florida-Cota), Malacoptila panamensis (La Fragua-Nilo), Amazona amazonica (cerca de Honda), Grallaricula flavirostris (MonterredondoGuayabetal), Sclerurus albigularis (Medina), Sublegatus arenarum? (Tibacuy), Parkesia motacilla (Parque La Florida-Cota) y Setophaga virens (Quebrada La Vieja-Bogotá). Otros registros son (Farallones de Medina): Aburri aburri, Ortalis ruficauda?, Bubo virginianus, Tachornis squamata, Ramphastos tucanus, Thectocercus acuticaudatus, Thamnophilus aethiops, Myrmophylax atrothorax, Myiarchus tyrannulus, Caccicus solitarius y Cacicus haemorrhous (Cortés et al., 2010); Buteogallus solitarius (Guayabetal) y Turdus fumigatus (Chuzalaguna de Fúquene) (http:/ /ipt.sibcolombia.net/ rnoa/resource.do?r=censo_aves_rnoa); Setopagis heterura, Siryster albocinereus, Stelgidopteryx serripennis y Turdus leucops (Sabana de Bogotá) (ABO, 2000); Myrmotherula ignota (Reserva Mana Dulce entre Agua de Dios-Nilo); Plegadis falcinellus (Cundinamarca) (Naranjo et al., 2012; León-Lleras y Rodríguez-Villamil, 2015), Chaetura chapmani (San Antonio del Tequendama) (Downing, 2005) y Cacicus haemorrhous (Medina) (Salaman et al., 2002). 
Tabla 2. Especies cuya única evidencia en el departamento corresponde a colecciones biológicas o registros sonoros. Con asterisco se encuentran las especies que requieren confirmación.

\begin{tabular}{|c|c|c|}
\hline & Especie & Localidad \\
\hline \multirow{29}{*}{ Colecciones biológicas } & Mustelirallus albicollis & \multirow{12}{*}{ Medina } \\
\hline & Chordeiles nacunda & \\
\hline & Jacamerops aureus & \\
\hline & Bucco macrodactylus & \\
\hline & Monasa flavirostris & \\
\hline & Bubucco richardsoni & \\
\hline & Epinecrophylla ornata & \\
\hline & Xiphorhynchus elegans & \\
\hline & Ochthornis littoralis & \\
\hline & Cotinga cayana & \\
\hline & Pachyramphus minor & \\
\hline & Turdus albicollis & \\
\hline & Vireolanius leucotis & Guayabetal \\
\hline & Charadrius collaris & \multirow{3}{*}{ Paratebueno } \\
\hline & Opisthocomus hoazin & \\
\hline & Hypnelus ruficollis & \\
\hline & Leptotila cassini & Puerto Salgar \\
\hline & Chloroceryle inda & \multirow{5}{*}{ Puerto Salgar } \\
\hline & Myrmotherula pacifica & \\
\hline & Gymnocichla nudiceps & \\
\hline & Tangara larvata & \\
\hline & Geothlypis aequinoctialis & \\
\hline & Pteroglossus torquatus & Puerto Salgar, Yacopí \\
\hline & Lepidothrix coronata & Girardot \\
\hline & Habia cristata & San Juan de Río Seco \\
\hline & Psarocolius wagleri & Apulo \\
\hline & Trogon rufus & \multirow{2}{*}{ Tena-Pedro Palo } \\
\hline & Quiscalus mexicanus & \\
\hline & Urosticte ruficrissa & Choachí \\
\hline
\end{tabular}


Cont. Tabla 2. Especies cuya única evidencia en el departamento corresponde a colecciones biológicas o registros sonoros. Con asterisco se encuentran las especies que requieren confirmación.

\begin{tabular}{lll}
\hline \multicolumn{1}{c}{ Especie } & \multicolumn{1}{c}{ Localidad } \\
\hline & \multicolumn{1}{c}{ Zentrygon frenata $*$} & Medina, H. Álvarez \\
\cline { 2 - 3 } Phaethornis longirostris & Yacopí, F. G. Stiles \\
\cline { 2 - 3 } & Anisognathus lacrymosus & \\
\cline { 2 - 3 } Registros sonoros & Cercomacra parkeri & $\begin{array}{l}\text { La Vega-Tabacal, O. } \\
\text { Cortés }\end{array}$ \\
\cline { 2 - 3 } & Platyrinchus coronatus & Fusagasugá, O. Laverde \\
\cline { 2 - 3 } & Megascops petersoni * & $\begin{array}{l}\text { San Antonio del } \\
\text { Tequendama, J. Bradley }\end{array}$ \\
\hline
\end{tabular}

Especies y subespecies extintas. Se reporta una especie extinta en Colombia, el zambullidor colombiano (Podiceps andinus) especie endémica de Colombia y de la cual se confirmó su extinción en 1982 (Fjeldså, 1993). En Cundinamarca fue registrada en la Sabana de Bogotá (laguna La Herrera, represa del Muña) y en la laguna de Fúquene (Olivares, 1969; Renjifo et al., 2016). Dos especies extintas en el departamento: Netta erythrophthalma registrada en la laguna de Fúquene y la Sabana de Bogotá (laguna La Herrera y Fontibón), su última observación fue en 1977 en Engativá y posiblemente se encuentre extinta en el país (Olivares, 1969; R. Ridgely en Hilty y Brown, 1986; Botero, 2002), y Macroagelaius subalaris registrada en Fusagasugá (última observación en 1960), Silvania, Subia, La Aguadita y Aguabonita (Olivares, 1969; Amaya-Espinel y Renjifo, 2002; Córdoba-Córdoba et al., 2014). Tres subespecies extintas: Anas georgica niceforoi, registrada en la laguna de Fúquene y en la Sabana de Bogotá (lagunas de La Herrera y Tibabuyes), considerada extinta desde 1956 (Olivares, 1969; Naranjo, 2002a), Anas cyanoptera borreroi registrada en la laguna de Fúquene y Cucunubá y en la Sabana de Bogotá (lagunas de La Herrera y Tibabuyes, Mosquera, Gachancipá, Torca y represa del Muña), no registrada desde los años 60s (Olivares, 1969; Naranjo, 2002b) y Polystictus pectoralis bogotensis registrada en la Sabana de Bogotá, pantanos de
Suba y cuyo último registro fue en 1919 (Olivares, 1969; Collar y Wege, 1995).

Distribución espacial de los registros en el departamento. Han sido estudiadas varias localidades a lo largo del departamento de Cundinamarca, las cuales se ubican en áreas restringidas principalmente al centro del departamento y en cercanías a la ciudad de Bogotá, presentando por ende grandes vacíos de información en sus límites. Hacia la zona occidental (límites con el valle del río Magdalena) hay pocos muestreos y estos se encuentran principalmente hacia la provincia del alto Magdalena (las provincias del Magdalena centro y bajo Magdalena son las que tienen menos información). Para la zona oriental, hacia la provincia de Medina (límites con los Llanos Orientales) hay muy pocos muestreos (Figura 7, Anexo 6). Estas dos regiones son las que presentan el mayor número de registros nuevos para el departamento debido a la falta de muestreo. Algo similar ocurre hacia el norte (provincias de Río Negro) y sur del departamento (provincias de Sumapaz y parte de Oriente y Distrito Capital) las cuales tienen muy pocas localidades muestreadas. Estas últimas principalmente deben ser exploradas al presentar la mayor cantidad de ecosistemas con áreas naturales como herbazales, arbustales y bosques del departamento (ver Figura 4). 


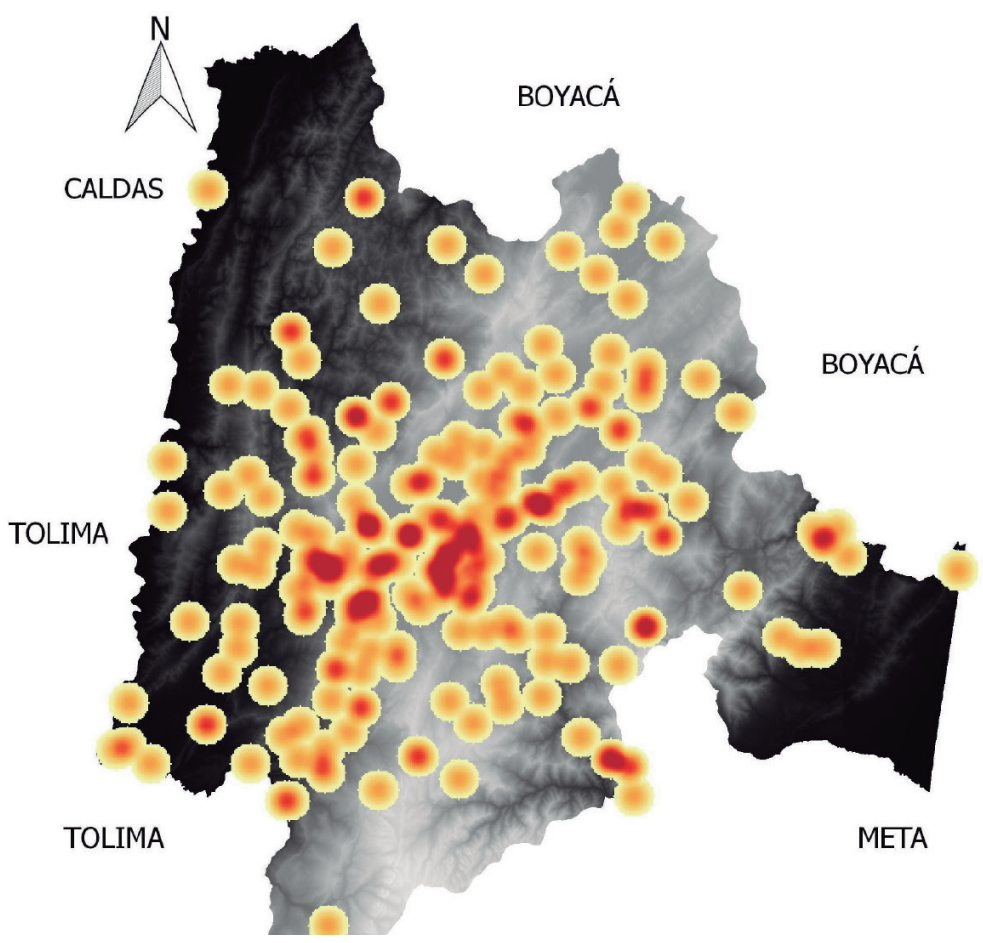

Figura 7. Mapa de densidad de Kernel de las localidades estudiadas históricamente en el departamento de Cundinamarca(Olivares, 1969; fuentes de información y observaciones de los autores). Los puntos rojos representan congregaciones de localidades estudiadas (no se tuvieron en cuenta listados aislados o con poca representatividad de muestreo).

Sin embargo, esto no significa que las localidades donde existe mayor número de estudios se encuentren inventariadas en su totalidad. Un claro ejemplo de esto es la región de Nilo y Tibacuy, donde en muestreos recientes realizados por algunos investigadores se han registrado nuevas especies para el departamento (Leptotila conoveri, Ara militaris, Malacoptila panamensis, Tiaris fuliginosus, Setophaga caerulescens) o de especies cuyos únicos registros han sido históricos (Phyllomyias burmeisteri) (González-Prieto et al., 2014; http://www.ebird.org; A. M. González datos no publicados).

Nuevos registros de especies para el departamento (otros autores). A continuación se realiza un recuento de las especies relevantes, ya sea por ser nuevos registros para el departamento y/o que han ampliado su rango de distribución o especies que presentan únicamente datos históricos:

-Anas bahamensis bahamensis: residente poco común a relativamente común en aguas salobres, áreas mareales y manglares, y menos frecuente en el interior en pantanos y lagunas de agua dulce (Hilty y Brown, 1986; Ruiz-Guerra y Estela, 2016). Presente en Colombia hasta los 100 m s.n.m. en la región Caribe desde Cartagena hasta La Guajira (Hilty y Brown, 1986; Ruiz-Guerra y Estela, 2016), con registros en Sinú-Córdoba (Ruiz-Guerra y Estela, 2016); puede ocurrir en terreno elevado de los Andes hasta los 2500 m s.n.m. y en ocasiones a altitudes superiores (Carboneras y Kirwan, 2016). Actualmente es catalogada 
como una especie Casi Amenazada (NT) debido principalmente a la destrucción del hábitat, la cacería y la contaminación (Renjifo et al., 2016). Fue observada en la laguna de La Herrera, municipio de Mosquera en 2015 (D. Rodríguez en prensa) y Anapoima (V. Vergara com. pers.), representando así los primeros registros para Cundinamarca y probablemente los primeros en el interior del país.

-Calidris fuscicollis: especie migratoria boreal poco común, presente en Colombia en Leticia (Amazonas), Puerto Inírida (Guainía), SFF Los Flamencos en La Guajira, PNN Sanquianga, isla Salamanca y Sabanagrande y SFF Malpelo hasta los $400 \mathrm{~m}$ s.n.m. en donde habita pastizales y playas arenosas, playones salinos y orillas de humedales salobres (Hilty y Brown, 1986; Ruiz-Guerra, 2012a). Registrada en la laguna de Chisacá en el PNN Sumapaz a 3700 m s.n.m. (A. Pinto datos no publicados). Este registro es el primero para la cordillera Oriental y el de mayor elevación conocida.

-Leptodon cayanensis cayanensis: presente en Colombia hasta los 1000 m s.n.m. en la costa Pacífica hacia el este por el norte de los Andes en el alto Sinú y Nechí (poco común); Caribe desde el Atlántico hasta la base oeste y noroeste de la Sierra Nevada de Santa Marta; sur en el valle del Magdalena hasta noroeste de Santander; este de los Andes desde el oeste de Caquetá y Vaupés en donde habita bosques húmedos y bordes de bosque (Hilty y Brown, 1986; Márquez et al., 2005). Fue fotografiada en Mámbita-Ubalá (N. León com. pers.) siendo el primer registro para Cundinamarca y representando una ampliación del rango de distribución en el país para el este de los Andes (posiblemente registrado en San Antonio del Tequendama, requiere confirmación).

-Morphnarchus princeps: es una especie que habita en Colombia en pendientes bajas y piedemontes en bosques húmedos entre los 50 y los 1800 m s.n.m. (principalmente 300-1200 m s.n.m.). Presente en la vertiente Pacífica desde Quibdó (Chocó) hacia el sur (valle del río Anchicayá, Valle del Cauca, PNN Munchique, reserva Tambito-Cauca), valle medio del río Magdalena (pendiente oeste de la cordillera
Oriental en Santander-Serranía de las Quinchas), cabeceras del río Magdalena en Huila (PNN Cueva de los Guácharos) y sector de Risaralda (Hilty y Brown, 1986; Laverde-R et al., 2005; http:/ / www. xeno-canto.org/). Fue registrada en el municipio de Guayabetal sector El Calvario-Monterredondo (A. Cuervo com. pers.), siendo el primer registro confirmado para el departamento y el primero para el este de los Andes.

-Buteo albigula: especie rara y local, habita bosques montanos húmedos, bosques de niebla y bosques achaparrados a mayores altitudes y zonas abiertas adyacentes a estos bosques entre los 1700 y 3500 m s.n.m.. Presente en Colombia en la cordillera Central (Quindío), valle del río Combeima, este de Popayán, noroeste del volcán Puracé, Munchique y El Tambo en el departamento del Cauca (Hilty y Brown, 1986; Márquez et al., 2005). Esta especie fue observada en el PNN Chingaza en 2015 (F. Gómez com. pers.) y en 2016 en la ciudad de Bogotá (J. Velásquez com. pers.) lo cual representa los primeros registros para Cundinamarca y amplían la distribución en el país.

-Megascops ingens (venezuelanus?): esta subespecie se distribuyen en Colombia entre los 1200 y 1700 m s.n.m. (hasta los 2250 m s.n.m.), sobre el flanco occidental de la Serranía del Perijá, La Guajira, Santander y Boyacá (Pajarito), M. i. ingens presente en los Andes al suroeste de Colombia hacia el sur (Hilty y Brown, 1986; ChaparroHerrera et al., 2015). Registrada y fotografiada en el municipio de Bojacá en 2016 (A. Bautista com. pers.), ampliando así considerablemente su distribución en el país además de ser el primer registro para Cundinamarca (Chaparro-Herrera, 2017) (registro sonoro en Medina por M. Álvarez, requiere confirmación).

-Nyctibius maculosus: es una especie muy rara que habita bosques nublados con claros entre los 1800 y los 2800 m s.n.m. Se conoce en Colombia únicamente de "pieles de Bogotá" y de la vertiente oeste de la cordillera Oriental en el Norte de Santander y vertiente este en Llorente en sureste de Nariño (Hilty y Brown, 1986; Cohn-Haft, 2016). 
Esta especie fue observada, fotografiada y grabada vocalmente en el PNN Chingaza en 2015 (A. Bautista datos no publicados), lo cual representa el primer registro para el departamento además de una nueva localidad, de las pocas que se conocen, en el país.

-Ara militaris militaris: es una especie Vulnerable (VU) y presenta migraciones locales (hasta los 2000 m s.n.m. o más). Habita selvas húmedas y vegetación subxerofítica (Santa Marta). Su distribución es discontinua en Colombia: Sierra Nevada de Santa Marta y cordillera Oriental desde la serranía de Perijá hacia el sur a lo largo de la vertiente occidental hasta Bucaramanga; vertiente oriental de la cordillera Oriental desde la Serranía de La Macarena hasta el Putumayo; vertiente oriental de la cordillera Central en Remedios y anteriormente en el área de Medellín y en la vertiente occidental de la cordillera Occidental cerca de las cabeceras del San Juan hasta Dagua y Anchicayá (Hilty y Brown, 1986; RodríguezMahecha, 2002). Fue observado un grupo de nueve individuos en sobrevuelo en el municipio de Nilo en 2015 (http://www.ebird.org). Este registro representa el primero para el departamento además de llenar un vacío de información en la distribución de la especie en el país.

-Furnarius leucopus endoecus: presente en Colombia en áreas húmedas y lodosas y en matorrales enmarañados a lo largo de cuerpos de agua (no siempre) y en zonas semiabiertas con suelo desnudo hasta los 500 m s.n.m., desde la serranía de San Jacinto y sur del Magdalena, por el sur hasta el alto río Sinú y valle medio del río Magdalena hasta Puerto Berrio y tierras bajas de la serranía de los Yariguíes (Hilty y Brown, 1986; Donegan et al., 2010; Remsen y de Juana, 2016). Registrada en el municipio de Beltrán (S. Lleras com. pers.), siendo el primero para Cundinamarca y la localidad más hacia el sur del valle del río Magdalena, ampliando su distribución hacia esta región.

-Chloropipo flavicapilla: especie casi-endémica y Vulnerable (VU) poco común, presente entre los 1200 y los 2400 m s.n.m., habita bosques húmedos y vegetación secundaria alta, puntualmente en ambas vertientes de la cordillera Occidental (Valle y Cauca), vertiente occidental de la cordillera Central (Antioquia) y Oriental (desde Tolima hasta Caldas y extremo norte en Antioquia), cabecera del valle del río Magdalena (Huila), y en el flanco occidental de la cordillera Oriental (Tolima) (Hilty y Brown, 1986; Moreno-Palacios, 2014). Fue observada en la laguna de Pedro PaloTena (D. Calderón datos no publicados) lo cual representa el primer registro para el departamento y para esta región del país.

- Piprites chloris: especie poco común presente en Colombia hasta los 1500 m s.n.m. en la serranía de Perijá, extremo noroeste de la cordillera Central, valle medio del Magdalena, este de los Andes desde el oeste de Meta hasta el sureste de Nariño, también registrada en Vaupés y sureste de Guainía en donde habita bosque húmedo y bosque secundario maduro (Hilty y Brown, 1986; Snow y de Juana, 2017). Fue grabado vocalmente en Medina (O. Laverde com. pers.), lo cual representa el primer registro para el departamento y la localidad más al norte en el flanco oriental de la cordillera Oriental.

- Setophaga caerulescens: especie migratoria boreal rara en Colombia, registrada únicamente en San Andrés y Providencia, La Guajira y Magdalena, con registros aislados en Santander y Quindío por debajo de los 2000 m s.n.m. (Hilty y Brown, 1986; Escrucería y Peraza, 2012a). Fue registrada en el cerro de Quininí (http:/ / www.ebird.org; A. Caguazango com. pers.) siendo el primer registro para el departamento y uno de los pocos al interior del país.

-Tiaris fuliginosus: es una especie no bien conocida en Colombia, aparentemente local o estacional poco común, habita matorral seco, en bordes de bosque húmedo o en claros con pastos en monte claro, posiblemente hasta los $1000 \mathrm{~m}$ s.n.m. (en Venezuela hasta los 2300 m s.n.m.), presente en el alto valle del río Magdalena y alto Patía, región de Santa Marta y Bucaramanga, posiblemente en otros lugares de manera estacional (Hilty y Brown, 
1986; Ridgely y Tudor, 2009). Especie capturada en el municipio de Nilo (A. M. González datos no publicados), lo cual representa el primer registro para el departamento y una ampliación del rango de distribución en el país.

\section{Nuevos registros de especies para el departamento con ampliación en su rango de distribución (registros de los autores):}

-Crypturellus cinereus: presente hasta los $500 \mathrm{~m}$ s.n.m. en la base este de la cordillera Oriental en el norte de Meta y el este de Vichada hacia el sur hasta Putumayo. Fue registrada en el municipio Ubalá (Mámbita) a 1200 m s.n.m. en el interior de un bosque secundario ampliando por ende hacia el norte su distribución y aumentando su rango de elevación conocido 500 m s.n.m. (registros recientes en Medina, O. Laverde, com. pers.).

-Syrigma sibilatrix fostersmithi: registrada en Colombia principalmente hasta los 500 m s.n.m. en el este de los Andes por el sur hasta el río Guaviare y por el este hasta el noroeste de Vichada (Hilty y Brown, 1986), sin embargo, existen algunos registros al oeste de los Andes (Parra-Hernández, et al.,2007). Fue registrada en el municipio de Ubalá a 815 m s.n.m. representando el primero para el departamento, sin embargo en febrero de 2016 en las Gravilleras de Siecha (Guasca) a 2650 m s.n.m. fueron observados dos individuos (J. J. Prada com. pers.), ampliando considerablemente su distribución altitudinal (registrada recientemete en el lago de Tota (Boyacá) a 3015 m s.n.m. por Zuluaga-Bonilla y Macana-García, 2016). Vale la pena resaltar que poblaciones de los Andes de Venezuela se encuentran a 2300 m s.n.m. y existen registros excepcionales en Bolivia a 2500 m s.n.m. (Martínez-Vilalta et al., 2007). También fue registrada recientemente en los municipios de Beltrán (S. Lleras com. pers.) y Guayabetal.

-Calidris bairdii: migratoria boreal que se distribuye en Colombia al oeste de los Andes hasta los 3400 m s.n.m.; registros en la zona Pacífica (PNN Gorgona, Sanquianga y SFF Malpelo), zona Caribe (Córdoba, La Guajira y Magdalena) Valle del Cauca, Antioquia, Cauca, Chocó y Nariño (Ruiz-Guerra 2012b). Registrado en el páramo de Sumapaz a 3700 m s.n.m. representando el primero para el departamento y el segundo para la cordillera Oriental (P. Kaestner lo registró en 1989 en el lago de Tota-Boyacá) (http://www. ebird.org).

-Buteo albonotatus: se encuentra distribuida en Colombia desde el Golfo de Urabá por la región Caribe hasta La Guajira y hacia el suroriente de Córdoba, sur del Magdalena y centro del Cesar con registros recientes en Antioquia y un registro en Pueblo Rico-Risaralda, al oriente de los Andes de manera dispersa y un registro histórico en el departamento de Huila, hasta los 500 m s.n.m. (a los 2400 m s.n.m. en la Sierra Nevada de Santa Marta) (Hilty y Brown, 1986; Márquez et al., 2005, Bierregaard et al., 2016); fue registrada en el municipio de Apulo siendo el primero para el departamento y ampliando su rango de distribución hacia el valle alto del río Magdalena (Chaparro-Herrera et al., 2017).

-Buteogallus urubitinga urubitinga: habita bordes de bosque, bosques ribereños y pantanosos, manglares y sabanas con parches de bosque hasta los 1500 m s.n.m. (raro a 1800 m s.n.m.) desde el límite con Panamá hacia el sur por el Pacífico hasta la serranía del Baudó y desde el golfo de Urabá, por el este por hasta la región Caribe hasta La Guajira, y toda la región este de los Andes (Hilty y Brown, 1986; Bierregaard et al., 2017). Registrado en el municipio de Apulo siendo el primer registro para el departamento y ampliando la distribución por el valle del río Magdalena hacia el sur.

-Phaethornis bourcieri bourcieri: presente en Colombia hasta los $400 \mathrm{~m}$ s.n.m. en el sureste de los Andes desde el oeste de Caquetá (sur de Florencia), noreste de Guainía (Puerto Inírida) y Vaupés (hasta los 1600 m s.n.m. en Venezuela) (Hilty y Brown, 1986; Hinkelmann et al., 2016). Esta especie fue registrada en el municipio de Ubalá (Mámbita) a 815 m s.n.m., representado el primero para el departamento y ampliando la distribución hacia el norte del este de los Andes. 
-Chlorestes notata notata: Es una especie distribuida hasta los 500 m s.n.m. al este de los Andes (probablemente todo el este) con unas pocas localidades dispersas conocidas (río Orinoco en Maipures, llanos del Casanare, y Leticia (C. $n$. puruensis)) (Hilty y Brown, 1986; McNish, 2007; Bündgen y Kirwan, 2016). Fue observada en el municipio de Ubalá (Mámbita) a los 1240 m s.n.m. siendo el primero para el departamento además de ampliar su rango altitudinal y aportando una nueva localidad para el este de los Andes.

-Atticora tibialis griseiventris: presente en el este de los Andes en la serranía de la Macarena, oeste de Caquetá, sureste de Nariño y Vaupés hasta los 1600 m s.n.m. (principalmente hasta los 1000 m s.n.m.) (Hilty y Brown, 1986; Turner y Kirwan, 2016). Fue registrada en el municipio de Ubalá (Mámbita) siendo el primer registro para el departamento y ampliando la distribución de la especie hacia el norte del este de los Andes.

- Hylocichla mustelina: especie migratoria boreal con pocos registros en el norte del país, Parque Nacional Natural Los Katíos, noreste del Chocó, islas de San Andrés y Providencia y La Guajira (Hilty y Brown 1986; Eusse-González, 2012). Registrada en el cerro de Quininí, municipio de Tibacuy (1400 m s.n.m.), ampliando considerablemente su rango de distribución y siendo a la fecha el primer registro confirmado para el interior del país.

\section{Especies relevantes cuyo único registro para el departamento se encuentra en Olivares (1969):}

-Anas crecca carolinensis: es una especie errante (http:/ / www.museum.lsu.edu/ Remsen/ SACCBaseline.html). Se anota que es uno de los registros más raros en Colombia y se conoce por dos ejemplares coleccionados en el municipio de Fúquene (laguna de Fúquene) en enero 24 (sin año).

-Gallinago imperialis: es una especie Casi Amenazada (NT) a nivel global (IUCN: http:// www.iucnredlist.org/) y categorizada como
Datos Deficientes (DD) a nivel nacional (Renjifo et al., 2016). Se distribuye entre los 2700 y los 3800 m s.n.m. a lo largo de la vertiente oriental del norte de los Andes desde Colombia y Ecuador hasta la cordillera de Vilcabamba al sur del Perú (extremadamente rara y local) (Renjifo et al., 2002; Renjifo et al., 2016). Se anota que en Colombia se conoce de dos pieles de "Bogotá", Nicéforo María registró provisionalmente especímenes del páramo de Choachí y de la Laguna de Siecha, pero este material se quemó el 9 de abril de 1948 ("El Bogotazo"). A su vez solo se cuenta con registros visuales de la década de 1980 en el Parque Nacional Natural Chingaza (Renjifo et al., 2016).

-Polytmus guainumbi andinus: presente en Colombia hasta los 600 m s.n.m. en la base este de la cordillera Oriental, por el sur hasta la serranía de la Macarena y por el este hasta el río Orinoco en Vichada (Hilty y Brown, 1986; McNish, 2007). Existen algunos registros al oeste de los Andes en el Parque Nacional Los Katios, Santander y Norte de Santander (hasta los 1300 m s.n.m.) (Avendaño et al., 2013). Se anota que el tipo es de "pieles de Bogotá", probablemente coleccionadas en la base de la ladera oriental en los Andes entre Cundinamarca y Meta (Medina).

-Discosura conversii: se encuentra en Colombia hasta los 1000 m s.n.m. (un registro a los 1400), en toda la costa Pacífica y hacia la serranía de las Quinchas (Otanche-Boyacá) (Hilty y Brown, 1986; Biomap: http://www.biomap.net). Se anota que existen indicios de que haya sido coleccionada en Yacopí.

-Chlorostilbon russatus: presente en Colombia entre los 600 y los 1700 m s.n.m. en la Sierra Nevada de Santa Marta y serranía de Perijá (Hilty y Brown, 1986). Se anota un registro en la laguna de Fúquene (2600 m s.n.m.) siendo el único registro que se tiene en el centro del país (coleccionado por K. von Sneidern en 1950).

-Laniocera hypopyrra: presente en Colombia hasta los 500 m s.n.m. en la base este de la cordillera Oriental desde el extremo suroriental de Casanare 
y noreste de Guainía (Puerto Inírida) hacia el sur (Hilty y Brown, 1986). Se anota que es una especie muy rara en colecciones científicas en cuanto se refiere a material colombiano y que los únicos registros auténticos del país son en Guaicaramo (Cundinamarca) y Los Micos (Meta).

\section{Especies relevantes representadas únicamen- te en colecciones científicas:}

-Urosticte ruficrissa: presente entre los 1600 y los 2300 m s.n.m. en la vertiente este de la cordillera Central en el sur del Huila (San Agustín) y sureste de Nariño (El Carmen) (Hilty y Brown, 1986; Ayerbe-Quiñones et al., 2008; Calderón-Leytón et al., 2011). Coleccionado en 1916 en el municipio de Choachí. Este registro representa el único para el departamento y amplía la distribución hacia el norte de la cordillera Oriental.

-Trogon rufus: presente hasta los 1100 m s.n.m. en la costa Pacífica y este por tierras bajas húmedas, norte de los Andes hasta valle medio del río Magdalena (sur hasta Honda-Tolima), subespecie sulphureus presente al este de los Andes (Hilty y Brown, 1986). Coleccionada en 1970 en la laguna de Pedro Palo en el municipio de Tena, siendo el único registro para el departamento. (T. $r$. cupreicauda?).

-Jacamerops aureus aureus: subespecie presente en Colombia al este de los Andes hasta los 900 m s.n.m. desde el sur del Meta (serranía de la Macarena) y sureste de Guainía hacia el sur hasta Nariño (Hilty y Brown, 1986; Calderón-Leytón et al., 2011). J. a. penardi presente en costa Pacífica y tierras bajas húmedas al norte de los Andes, por el este hasta el valle medio del río Magdalena (noroeste de Santander). Coleccionada en Medina, sin fecha, amplía su rango de distribución hacia el norte del este de los Andes.

-Vireolanius leucotis: presente en Colombia entre los 300 y los $2100 \mathrm{~m}$ s.n.m. (raro por encima de los 1800) en la vertiente Pacífica desde el alto río San Juan (cerro Tatamá) hacia el sur (V. $l$. mikettae) y base este de la cordillera Oriental en el sureste de Nariño (V. l. leucotis) (Hilty y Brown, 1986). Coleccionada en Quetame-Guayabetal en 1970, ampliando considerablemente su rango de distribución hacia el norte en la vertiente este de la cordillera Oriental (V. l. leucotis?).

-Habia cristata: es una especie endémica de Colombia, se encuentra distribuida entre los 700 y los 1800 m s.n.m. en la vertiente Pacífica, localmente en la vertiente este adyacente de la cordillera Occidental desde el norte de Antioquia hasta el Cauca y vertiente este de la cordillera Central en el Líbano e Ibagué-Tolima (Hilty y Brown, 1986; Biomap: http://www.biomap.net, Freeman et al., 2012; http://www.xeno-canto. org/). Coleccionado en San Juan de Río Seco en 1923, siendo el primer registro para la cordillera Oriental.

-Psarocolius wagleri ridgwayi: presente en Colombia hasta los 1000 m s.n.m. (principalmente menos de 400 m s.n.m.) en la costa Pacífica y tierras bajas húmedas al norte de los Andes este hasta valle medio del río Magdalena (sur hasta Remedio al este de Antioquia) (Hilty y Brown, 1986). Coleccionado en Apulo en 1975 siendo el único registro para el departamento. También ha sido registrada, actualmente, en la serranía de los Yariquíes (Santander) (Donegan et al., 2010).

-Quiscalus mexicanus: presente en la costa Caribe y en la costa Pacífica (Hilty y Brown, 1986). Coleccionado en 1970 en la laguna de Pedro Palo, municipio de Tena. Ese registro representa el primero hacia el interior del país y por ende amplia el rango de distribución de la especie. ( $Q$. m. peruvianus?)

\section{Otras especies de relevancia representes en el departamento por diferentes fuentes de información:}

-Plegadis falcinellus: especie migratoria boreal registrada en Colombia entre los 300 y los 2600 $\mathrm{m}$ s.n.m. (principalmente entre los 300 y los 1500 m s.n.m.) en los llanos de la Orinoquia y el Caribe (registros en Valle del Cauca, Caldas y 
Cundinamarca) (Naranjo et al., 2012, León-Lleras y Rodríguez-Villamil 2015). Para Cundinamarca son pocos los registros y se centran en la Sabana de Bogotá (2600 m s.n.m.) siendo el rango altitudinal más alto conocido para la especie en el país; registrado en el humedal el Tandil entre CajicáZipaquirá y en la laguna de Fúquene (McNish, 2010; León-Lleras y Rodríguez-Villamil, 2015).

-Platalea ajaja: presente en Colombia hasta los 1000 m s.n.m. en la costa Caribe desde Cartagena hasta el este de La Guajira, errante hasta el alto valle del Cauca en Cali (una vez en la costa Pacífica, Guapi), bajo valle del río Magdalena y este de los Andes (Hilty y Brown, 1986). Fue registrada en la Reserva Mana Dulce entre Agua de Dios-Nilo en 2010 por David Parsley (http://www.ebird.org), lo cual representa una ampliación de la distribución hacia la parte sur del valle del Magdalena.

-Buteogallus solitarius solitarius: especie amenazada globalmente, registrada entre los 200 y los $2200 \mathrm{~m}$ s.n.m., conocida al este de los Andes únicamente en el departamento de Caquetá (Florencia y Morelia) y un registro reciente en Boyacá (Santa María) (Hilty \& Brown, 1986; Márquez et al., 2005; ChaparroHerrera y Laverde, 2014). Existe un registro a su vez en Guayabetal (http://ipt.sibcolombia.net/ rnoa/resource.do?r=censo_aves_rnoa), el cual es importante mencionarlo por ser el primero para el departamento y debido a que a lo largo de su distribución se han confirmado menos de cien registros en los últimos ciento cincuenta años, por lo tanto es una de las rapaces menos conocidas (Phillips, 2012).

-Burhinus bistriatus pediacus: habita en Colombia sabanas secas y pastizales planos abiertos con o sin árboles dispersos y arbustos hasta los 500 m s.n.m. (hasta los 900 m s.n.m. en otros países), presente en la costa Caribe desde el área de Cartagena hasta La Guajira, valle del río Magdalena (alto valle, Ibagué-Tolima y Villavieja-Huila), valle alto del río Cauca y en general en el este de los Andes en los llanos hasta el río Guaviare (Hilty y Brown, 1986; McNish, 2007; Parra-Hernández et al., 2007; Ayerbe-Quiñones et al., 2008; Gómez y Robinson,
2014; Hume y Kirwan, 2016). Registrada en el municipio de Gutiérrez por Mayorca y Melo (2014) y Beltrán (S. Lleras com. pers.), siendo los primeros registros para Cundinamarca y en el segundo caso la segunda localidad en el Magdalena medio.

-Leucophaeus pipixcan: especie migratoria boreal poco común, registrada en Colombia en la costa Pacífica en las rocas de Octavia (Chocó), bahía de Buenaventura y Punta Soldado (Valle del Cauca), PNN Gorgona (Cauca) y Tumaco (Nariño) en donde habita playas arenosas, bajos areno-fangosos, manglares, también lagunas y ríos de interior (Hilty y Brown, 1986; Zamudio, 2012). Registrada (un inmaduro) en el Parque Metropolitano Simón Bolívar de Bogotá en 2011, siendo el primer registro para Cundinamarca y posiblemente el primero para la cordillera Oriental (Bayly, 2015).

-Leptotila conoveri: especie endémica presente entre los 1500 y los 2800 m s.n.m. en la vertiente este de la cordillera Central desde Ibagué por el sur hasta cabeceras del valle del río Magdalena en Huila y Cauca (Hilty y Brown, 1986; Parra-Hernández et al., 2007; Ayerbe-Quiñones et al., 2008). Recientemente registrada en el departamento de Cundinamarca en los municipios de Nilo y Tibacuy (GonzálezPrieto, 2014), siendo el primer registro para el departamento y para el este de los Andes.

-Megascops petersoni: presente entre los 1400 y 1820 m s.n.m. en el piedemonte de la cordillera Central en Anorí (Antioquia) y cordillera Oriental en la parte alta del municipio de San Luis de Cubarral (Cuervo et al., 2008; Chaparro et al., 2015; http:/ / www.xeno-canto.org/). Recientemente fue registrada auditivamente en el municipio de San Antonio del Tequendama (http://www.xenocanto.org/), ampliando su rango de distribución en la cordillera Oriental y siendo la única evidencia de su posible presencia en el departamento, sin embargo este registro requiere confirmación.

-Setopagis heterura: presente en Colombia hasta los 300 m s.n.m. en la base norte de la Sierra Nevada de Santa Marta y tierras bajas del Catatumbo 
(Cúcuta) (Hilty y Brown, 1986). Registrado a 2600 m s.n.m. en la Sabana de Bogotá (accidental) (ABO, 2000). Este registro amplia de manera considerable su rango de distribución y su rango altitudinal.

-Chaetura chapmani: presente en Colombia entre los 100 y los 1600 m s.n.m. en Antioquia, límite entre Risaralda y Antioquia (alto de Pisones), Risaralda, Valle del Cauca y en la cuenca del río Caquetá (Hilty y Brown, 1986; Ayerbe-Quiñones et al., 2008; Cuervo et al., 2003). Registrado en el municipio de San Antonio del Tequendama (Downing, 2005), representando el primer registro para el este de los Andes.

-Malacoptila panamensis magdalenae: subespecie presente hasta los $1400 \mathrm{~m}$ s.n.m. al lado oeste del golfo de Urabá por el este hasta las tierras bajas húmedas del norte de los Andes hasta valle medio del río Magdalena y sur hasta RemediosAntioquia. M. p. panamensis en la costa Pacífica en Chocó y M. p. poliopis en la costa Pacífica en el Valle por el sur hasta Nariño (Hilty y Brown, 1986; Rasmussen y Collar, 2016). Fue registrada por varios observadores en La Fragua-Nilo a los 1400 m s.n.m. (http:/ / www.ebird.org) ampliando levemente su distribución hacia la parte sur del valle medio del río Magdalena.

-Siryster albocinereus: presente en el este de Colombia hasta los 800 m s.n.m. a lo largo del flanco oriental de la cordillera Oriental (Donegan, 2013). Registrada en la Sabana de Bogotá (accidental) (ABO, 2000). Este registro amplía de manera considerable su rango altitudinal y su rango de distribución.

-Thamnophilus aethiops wetmorei: subespecie presente en Colombia hasta los 500 m s.n.m. (ocasionalmente a los 1150) en el este de los Andes desde la serranía de la Macarena hasta el oeste de Caquetá y Vaupés; este del Vichada (polionotus) y sureste de Nariño (aethiops) (Hilty y Brown, 1986; Zimmer e Isler, 2016a). Registrada en Farallones de Medina (Cortés et al., 2010), ampliando su distribución hacia el norte del este de los Andes en el pie de monte.
-Myrmotherula ignota ignota: subespecie presente hasta los 900 m s.n.m. en el oeste y norte de Colombia (por la vertiente Pacífica hacia el norte a lo largo de la base de los Andes por el este hasta Santander) (Zimmer e Isler, 2016b). Registrada en la Reserva Mana Dulce entre Agua de Dios y Nilo (Gómez y Robinson, 2014), ampliando su rango de distribución hacia el sur del valle del Magdalena.

-Vireo altiloquus: especie migratoria boreal poco común, presente en Colombia hasta 1200 m s.n.m. en las islas de San Andrés y Providencia, región de Santa Marta, La Guajira y Sucre por el sur hasta extremo norte de la cordillera Oriental (Santander) con registros aislados en Amazonas, Puerto Inírida, Guainía y Bogotá (en esta última localidad fue registrada en 1971) (T. B. Johnson en Hilty y Brown, 1986; Fierro-Calderón 2012) y coleccionada en 2012 en la Universidad Nacional de Colombia al estrellarse contra una ventana, siendo a la fecha los únicos registros presentes en el departamento de Cundinamarca y la localidad a mayor elevación (2600 m s.n.m.).

-Stelgidopteryx serripennis fulvipennis: especie migratoria registrada en Colombia únicamente en la laguna de Sonso (Valle del Cauca) (Downing, 2005) y un registro en la Sabana de Bogotá, registrada como accidental-desviada de su ruta de migración normal (ABO, 2000), datos que a su vez confirman su presencia en Suramérica.

-Turdus fumigatus: presente entre los 100 y los 1400 m s.n.m. al este de los Andes, T.f. aquilonalis en el noreste en Norte deSantandery T.f. orinocensis en el este (Hilty y Brown, 1986; Collar, 2017). Registrada en la laguna de Fúquene (2600 m s.n.m.) (http:/ / ipt.sibcolombia.net/rnoa/resource.do? $r={ }_{\text {censo }}$ aves_rnoa), ampliado considerablemente su rango altitudinal y siendo el primer registro para la parte alta de la cordillera Oriental.

-Turdus leucops: registrada entre los 1300 y los 2000 m s.n.m. en varias localidades a lo largo de las tres cordilleras. Presente en la cordillera Oriental en la serranía de los Yariguíes, vertiente este de la cordillera Oriental en el Meta y la serranía 
de la Macarena y serranía de Perijá también en el alto Magdalena en Huila (Hilty y Brown, 1986; Donegan et al., 2010; López-O et al., 2014). Registrada a su vez a los 2600 m s.n.m. en la quebrada la Vieja-Bogotá (ABO, 2000), ampliando su rango altitudinal y siendo el primer registro para la Sabana de Bogotá y para el departamento.

-Parkesia motacilla: especie migratoria boreal presente principalmente por debajo de los 2000 m s.n.m. al norte Colombia en la Sierra Nevada de Santa Marta; registros en el Valle del Cauca (Valle del Dagua) y en el Meta (Villavicencio) (Hilty y Brown, 1986; Escrucería y Peraza, 2012b). Registrado por P. Kaestner en 1989 en el Parque La Florida-Cota (2600 m s.n.m.) (http://www. ebird.org), siendo el primer registro para la Sabana de Bogotá y ampliando su rango de distribución altitudinal.

-Setophaga virens: especie migratoria boreal registrada en San Andrés y Providencia, vertiente norte de la Sierra Nevada de Santa Marta (cuchilla de San Lorenzo y Valparaíso), cordillera Oriental cerca de Bogotá y Valle de Aburrá (Hilty y Brown, 1986; Cifuentes-Sarmiento, 2012). Recientemente registrada (2009) por N. Bayly en la quebrada La Vieja-Bogotá (http://www.ebird.org), registro que confirma su presencia en la Sabana de Bogotá.

-Cacicus haemorrhous haemorrhous: presente hasta los 500 m s.n.m. en el este de los Andes desde el sur del Meta en la serranía de la Macarena y Vaupés (Mitú) hacia Venezuela (Hilty y Brown, 1986). Registrada en Medina (Salaman et al., 2002; Cortés et al., 2010), ampliando su distribución hacia el norte del este de los Andes en el pie de monte.

\section{Conclusiones}

Cundinamarca es uno de los departamentos con mayor riqueza de aves en Colombia, en parte gracias a la variación de sus condiciones geográficas y climáticas que implican heterogeneidad en los ecosistemas, permitiendo hábitats adecuados para el establecimiento de la fauna; hasta la fecha cuenta con 941 especies de aves, algunas migrantes latitudinales (101 especies) y se posiciona como región de importancia para la conservación de aves en el país al presentar 24 especies endémicas, 72 casi endémicas y 39 amenazadas. La revisión de los registros históricos y actuales ofrece nociones de dinámicas poblacionales y eventos de extinción y colonización en el departamento. Sin embargo no se cuenta con una caracterización completa; si bien son numerosas las localidades que han sido estudiadas, existen grandes vacíos de información en los límites departamentales, principalmente hacia el valle del río Magdalena y hacia la provincia de Medina en cercanía con los Llanos Orientales. Por lo anterior es importante llevar a cabo inventarios, estudios ecológicos (en diferentes épocas para dar cuenta de los cambios en el tiempo) y sistematización de datos en algunas zonas, especialmente aquellas que han sido poco exploradas y donde el recambio de especies puede ser elevado dadas las variaciones ambientales, geográficas y bióticas. Esto aportaría información relevante que puede llenar vacíos de información de distribución, historia natural y ecología, y posibilitar planes de conservación y manejo tanto de las especies como de sus hábitats.

\section{Agradecimientos}

Agradecemos a la Fundación Humedales, Fundación Ecotrópico Colombia, Asociación Bogotana de Ornitología-ABO; a L. Raz y H. Agudelo del Instituto de Ciencias Naturales de la Universidad Nacional de Colombia; a A. Cuervo, A. Marín, A. Pinto, D. Rodríguez, F. Gómez, J. P. Gómez, M. Álvarez, M. Hernández, N. León, S. Lleras y O. Laverde por la información suministrada, así como a tres revisores anónimos, $\mathrm{y}$ en general a todos los observadores de aves y ornitólogos por compartir sus registros en diferentes plataformas. Finalmente SCH agradece a C. J. Chaparro Jiménez (Q.E.P.D) por su compañía y apoyo. 


\section{Referencias}

Asociación Bogotana de Ornitología (ABO). (2000). Aves de la Sabana de Bogotá, guía de campo (276 pp). Bogotá D. C.: Asociación Bogotana de Ornitología y Corporación Autónoma Regional de Cundinamarca CAR.

Amaya-Espinel, J. D. y Renjifo, L. M. (2002). Macroagelaius subalaris. En Renjifo, L. M., FrancoMaya, A. M., Amaya-Espinel, J. D., Kattan, G. H. y López-Lanús, B. (Eds.). Libro rojo de aves de Colombia. Pp. 470-472 . Serie libros rojos de especies amenazadas de Colombia. Bogotá D. C.: Instituto de Investigación de Recursos Biológicos Alexander von Humboldt y Ministerio del Medio Ambiente.

Avendaño, J. E., Cortés-Herrera, J. O., BriceñoLara, E. R. y Rincón-Guarín, D. A. (2013). Crossing or bypassing the Andes: a commentary on recent range extensions of cis-Andean birds to the West of the Andes of Colombia. Orinoquia, 17(2), 207-214.

Avendaño, J. E., Bohórquez, E. I., Rosselli L., Arzuza-Buelvas D., Estela, F. A., Cuervo, A. M., Stiles, F. G. y Renjifo, L. M. (2017). Lista de chequeo de las aves de Colombia: una síntesis del estado del conocimiento desde Hilty \& Brown (1986). Ornitología Colombiana, 16:eA01.

Ayerbe-Quiñones, F., López-Ordóñez, J. P., González-Rojas, M. F., Estela, F. A., RamírezBurbano, M. B., Sandoval-Sierra, J. V. y GómezBernal, L. G. (2008). Aves del departamento del Cauca-Colombia. Biota Colombiana, 9(1), 77-132.

Bayly, N. (2015). Primer registro de la Gaviota de Franklin (Leucophaeus pipixcan) en la cordillera Oriental de Colombia. Boletín SAO, 24 (1y2), 1314.

Bierregaard, R. O., Boesman, P. y Kirwan, G. M. (2016). Great Black Hawk (Buteogallus urubitinga). En del Hoyo, J., Elliott, A., Sargatal, J., Christie, D. A. y de Juana, E. (Eds.). Handbook of the Birds of the World Alive. Barcelona: Lynx Edicions. Recuperado de: http://www.hbw. com/node/53113. Revisado el 1 de febrero 2017.
Bierregaard, R. O., Kirwan, G. M. y Marks, J. S. (2016). Zone-tailed Hawk (Buteo albonotatus). En del Hoyo, J., Elliott, A., Sargatal, J., Christie, D. A. y de Juana, E. (Eds.). Handbook of the Birds of the World Alive. Barcelona. Lynx Edicions. Recuperado de: http://www.hbw.com/ node/53132. Revisado el 21 de septiembre de 2016.

Botero, J. E. (2002). Oxyura jamaicensis. En Renjifo, L. M., Franco-Maya, A. M., Amaya-Espinel, J. D., Kattan, G. H. y López-Lanús, B. (Eds.). Libro rojo de aves de Colombia. Serie libros rojos de especies amenazadas de Colombia (104-108 pp). Bogotá D. C.: Instituto de Investigación de Recursos Biológicos Alexander von Humboldt y Ministerio del Medio Ambiente.

Bündgen, R. y Kirwan, G. M. (2016). Blue-chinned Emerald (Chlorostilbon notatus). En del Hoyo, J., Elliott, A., Sargatal, J., Christie, D. A. y de Juana, E. (Eds.). Handbook of the Birds of the World Alive. Barcelona. Lynx Edicions. Recuperado de http://www.hbw.com/node/55435. Revisado el 18 de febrero 2016.

Calderón-Leytón, J. J., Flórez, C., Cabrera-Finley, A. y Rosero, Y. (2011). Aves del departamento de Nariño, Colombia. Biota Colombiana, 12(1), 31-116.

Carboneras, C. y Kirwan, G. M. (2016). Whitecheeked Pintail (Anas bahamensis). En del Hoyo, J., Elliott, A., Sargatal, J., Christie, D. A. y de Juana, E. (Eds.). Handbook of the Birds of the World Alive. Barcelona. Lynx Edicions. Recuperado de: http://www.hbw.com/node/52886. Revisado el 17 de febrero 2016.

Castro, L. F., Ortega D. y Chaparro-Herrera S. (2015). Aves de la vereda Palo Blanco, municipio de Bituima-Cundinamarca, Colombia. The Field Museum-Rapid Color Guide, 653.

Chaparro-Herrera, S. (2017). Búhos del departamento de Cundinamarca, Colombia. Spizaetus, 22-28.

Chaparro-Herrera, S., Lopera-Salazar, A., Cediel, F. y Davies, R. (2017). Distribución del Águila 
Gallinaza (Buteo albonotatus) en Colombia y nuevos registros en el valle alto y medio del río Magdalena. Cotinga, 39, 78-81.

Chaparro-Herrera, S. (2015). Avifauna registrada en 14 Parques Ecológicos Distritales de Humedal (PEDH) de Bogotá. En Chaparro-Herrera, S. y Ochoa, D. (Eds). Aves de los humedales de Bogotá, aportes para su conservación (68-91 pp). Bogotá D. C.: Asociación Bogotana de Ornitología (ABO).

Chaparro-Herrera, S., Echeverry-Galvis, M. Á., Córdoba-Córdoba, S. y Sua-Becerra,A. (2013). Listado actualizado de las aves endémicas y casiendémicas de Colombia. Biota Colombiana, 14(2), 235-272.

Chaparro-Herrera, S., Córdoba-Córdoba S., LópezOrdóñez, J. P., Restrepo Cardona, J. S. y CortesHerrera, O. (2015). Los búhos de Colombia (271323). En Enríquez, P. (Ed.). Los búhos neotropicales: diversidad y conservación. México. Ecosur.

Chaparro-Herrera, S. y Laverde, O. (2014). Nueva localidad para el Águila Solitaria (Buteogallus solitarius) en Colombia. Boletín SAO, 23, (1-2): 1517.

Cifuentes-Sarmiento, Y. (2012). Setophaga virens. En Naranjo, L. G., Amaya, J. D., Eusse-González, D. y Cifuentes-Sarmiento, Y. (Eds.). Guía de las especies migratorias de la biodiversidad en Colombia. Aves (568-570 pp). Vol. 1. Bogotá D.C.: Ministerio de Ambiente y Desarrollo Sostenible/WWF Colombia.

Cohn-Haft, M. (2016). Andean Potoo (Nyctibius maculosus). En del Hoyo, J., Elliott, A., Sargatal, J., Christie, D. A. y de Juana, E. (Eds.). Handbook of the Birds of the World Alive. Barcelona. Lynx Edicions. Recuperado de: http://www.hbw. com/node/55157. Revisado el 17 de febrero 2016.

Collar, N. (2017). Cocoa Thrush (Turdus fumigatus). En del Hoyo, J., Elliott, A., Sargatal, J., Christie, D. A. y de Juana, E. (Eds.). Handbook of the Birds of the World Alive. Barcelona. Lynx Edicions. Recuperado de: http://www.hbw.com/ node/58299. Revisado el 1 de febrero 2017.
Collar, N. I. y Wege, D. C. (1995). The distribution and conservation status of the Bearded Tachuri Polystictus pectoralis. Bird Conservation International, 5, 367-390.

Córdoba, S. y Ahumada, J. (2005). Confirmation of Buff-fronted Owl Aegolius harrisii for the cordillera Oriental of Colombia. Bulletin of the British Ornithologists' Club, 125 (1), 56-58.

Córdoba-Córdoba, S., Cortés-Herrera O. y Hernández-Jaramillo A. (2014). Macroagelaius subalaris. En Renjifo, L. M., Gómez, M. F., Velásquez-Tibatá, J., Amaya-Villarreal, A. M., Kattan, G. H., Amaya-Espinel, J. D. y BurbanoGirón, J. (Eds.). Libro rojo de aves de Colombia, Volumen I: bosques húmedos de los Andes y la costa Pacífica (352-355 pp). Bogotá D.C.: Editorial Pontificia Universidad Javeriana e Instituto de Investigación de Recursos Biológicos Alexander von Humboldt.

Córdoba, S., Echeverry, M. Á., Umaña, A. M., Quintero, I., Iguera, M., Prieto, A., Mendoza, H., Villarreal, H. y Rengifo, J. M. (2007). Caracterización de la biodiversidad de cuatro áreas del Parque Nacional Natural Chingaza, Cundinamarca, Colombia (Informe técnico) (187 pp). Bogotá D. C.: Instituto de Investigación de Recursos Biológicos Alexander von Humboldt.

Cortés, F., Pinzón, V., Pulido, S., Cortés, O., López, J. y Roa, A. (2010). Assessment and Conservation of Cundinamarca Antpitta (Grallaria kaestneri) at Farallón de Medina, Cundinamarca (Informe técnico) (50 pp). Bogotá D. C.: Conservation Leadership Programme.

Cuéllar, D. 2011. Ambiente y desarrollo en el departamento de Cundinamarca, año 2005 (Trabajo de grado) (166 pp) . Bogotá D.C.: Universidad Nacional de Colombia, facultad de Ciencia Económicas, Instituto de Estudios Ambientales (IDEA), maestría en Medio Ambiente $\mathrm{y}$ Desarrollo.

Cuervo, A. M., Pulgarín, P. y Calderón, D. (2008). New distributional bird data from the Cordillera Central of the Colombian Andes, with implications for the biogeography of 
northwestern South America. The Condor, 110(3), 526-537.

Cuervo, A. M., Stiles F. G., Cadena C. D., Toro J. L. y Londoño. (2003). New and noteworthy bird records from the northern sector of the Western Andes of Colombia. Bulletin of the British Ornithologists' Club, 123(1), 7-24.

Devenish, C., Díaz, D. F., Clay, R. P., Davidson, I. y Yépez, I. (Eds). (2009). Important Bird Areas Americas-Priority sites for biodiversity conservation. BirdLife Conservation Series, 16.

Donegan, T. (2013). Vocal variation and species limits in the genus Sirystes (Tyrannidae). Conservación Colombiana, 19, 11-30.

Donegan, T. y Avendaño, E. (2010). A new subspecies of mountain tanager in the Anisognathus lacrymosus complex from the Yariguíes Mountains of Colombia. Bulletin of the British Ornithologists' Club, 130(1), 13-32.

Donegan, T. M., Avendaño, J. E., Briceño-L., E. R., Luna, J. C., Roa, C., Parra, R., Turner, C.,. Sharp, M. y Huertas, B. (2010). Aves de la serranía de los Yariguíes y tierras bajas circundantes, Santander, Colombia. Cotinga, 32, 72-89.

Downing, C. (2005). New distributional information for some Colombian birds, with a new species for South America. Cotinga, 24, 13-15.

Escrucería, S. y Peraza, C. (2012a). Setophaga caerulescens. En Naranjo, L. G., Amaya, J. D., Eusse-González, D. y Cifuentes- Sarmiento, Y. (Eds.). Guía de las especies migratorias de la biodiversidad en Colombia. Aves (562-564 pp). Vol. 1. Bogotá D.C.: Ministerio de Ambiente y Desarrollo Sostenible/WWF Colombia.

Escrucería, S. y Peraza, C. (2012b). Parkesia moracilla. En Naranjo, L. G., Amaya, J. D., EusseGonzález, D. y Cifuentes- Sarmient, Y. (Eds.). Guía de las Especies Migratorias de la Biodiversidad en Colombia. Aves (591-593 pp). Vol. 1. Bogotá D.C.: Ministerio de Ambiente y Desarrollo Sostenible/WWF Colombia.

Eusse-González, D. (2012). Hylocichla mustelina. En Naranjo, L. G., Amaya, J. D., Eusse-González,
D. y Cifuentes- Sarmiento, Y. (Eds.). Guía de las Especies Migratorias de la Biodiversidad en Colombia. Aves. Vol. 1 (486-488 pp). Bogotá D.C.: Ministerio de Ambiente y Desarrollo Sostenible/ WWF Colombia.

Fierro, K. (2009). Aves migratorias en Colombia. En Naranjo, L. G. y Amaya-Espinel, J. D. (Eds.). Plan Nacional de las Especies Migratorias: Diagnóstico e identificación de acciones para la conservación y el manejo sostenible de las especies migratorias de la biodiversidad colombiana (63-76 pp). Bogotá D.C.: Ministerio de Ambiente, Vivienda y Desarrollo Territorial y WWF Colombia.

Fierro-Calderón, E. (2012). Vireo altiloquus. En Naranjo, L. G., Amaya, J. D., Eusse-González, D. y Cifuentes- Sarmiento, Y. (Eds.). Guía de las especies migratorias de la biodiversidad en Colombia. Aves (455-457 pp). Bogotá D.C.: Vol. 1. Ministerio de Ambiente y Desarrollo Sostenible/WWF Colombia.

Franco, A. M. y Bravo, G. (2005). Áreas importantes para la conservación de las aves en Colombia. En BirdLife International y Conservación International. (Eds.). Áreas importantes para la conservación de las aves en los Andes tropicales: sitios prioritarios para la conservación de la biodiversidad (117-132). BirdLife Conservation Series, 14.

Franco, A. M., Amaya-Espinel, J. D., Umaña, A. M., Baptiste, M. P. y Cortés, O. (Eds). (2009). Especies focales de aves de Cundinamarca: estrategias para la conservación (144 pp). Bogotá D. C.: Instituto de Investigación de Recursos Biológicos Alexander von Humboldt y Corporación Autónoma Regional de Cundinamarca.

Fjeldså, J. (1993). The decling and probably extinction of the Colombian Grebe Podiceps andinus. Bird Conservation International, 3, 221234.

Freeman, B. G., Hilty S. L., Calderón-F, D., Ellery, T. y Urueña, L. E. (2011). New and noteworthy bird records from central and northern Colombia. Cotinga, 34, 5-16.

Gómez, J. P. y Robinson, S. K. (2014). Aves del bosque seco tropical de Colombia: las 
comunidades del valle alto del río Magdalena. En Pizano, C y H. García. (Eds.). El bosque seco tropical en Colombia (Pp. 95-127). Bogotá D.C.: Instituto de Investigación de Recursos Biológicos Alexander von Humboldt (IAvH).

González-Prieto, A., Espejo, N., Sanabria-Mejía, J., González-Prieto, C., Cormier, D. y Hobson, K. (2014). First record of Tolima Dove Leptotila conoveri in the Colombian East Andes. Bulletin of the British Ornithologists' Club, 134 (4), 307-310.

Graves, G. R. (1993). Relict of a lost world: A new species of Sunangel (Trochilidae: Heliangelus) from Bogotá. The Auk, 110, 1-8.

Hilty, S. L. y Brown, W. L. (1986). A guide to the birds of Colombia. New Jersey, USA: Princeton University Press, Princeton. 836 pp.

Hinkelmann, C., Kirwan G. M. y Boesman P. (2016). Straight-billed Hermit (Phaethornis bourcieri). En del Hoyo, J., Elliott, A., Sargatal, J., Christie, D. A. y de Juana, E. (Eds.). Handbook of the Birds of the World Alive. Barcelona: Lynx Edicions. Recuperado de http://www.hbw. com/node/55364. Revisado el 18 de febrero 2016.

Hume, R. y Kirwan, G. M. (2016). Double-striped Thick-knee (Burhinus bistriatus). En del Hoyo, J., Elliott, A., Sargatal, J., Christie, D. A. y de Juana E. (Eds.). Handbook of the Birds of the World Alive. Barcelona: Lynx Edicions. Recuperado de http://www.hbw.com/node/55364 Revisado el 18 de febrero 2016.

Instituto de Ciencias Naturales (ICN). (2015). Base de datos colección de Aves del Instituto de Ciencias Naturales (ICN). Bogotá D.C.: Universidad Nacional de Colombia.

Ideam. (2007). Ecosistemas continentales, costeros y marinos de Colombia. Bogotá D. C.: Instituto de Hidrología, Meteorología y Estudios Ambientales (Ideam), Instituto Geográfico Agustín Codazzi (IGAC), Instituto de Investigación de Recursos Biológicos Alexander von Humboldt (IAvH), Instituto de Investigaciones Ambientales del Pacífico Jhon von Neumann
(IIAP), Instituto de Investigaciones Marinas y Costeras José Benito Vives De Andréis (Invemar) e Instituto Amazónico de Investigaciones Científicas (SINCHI). Pp. $276+37$ hojas cartográficas.

Laverde-R., O., Stiles, F. G. y Múnera-R., C. (2005). Nuevos registros e inventario de la avifauna de la serranía de las Quinchas, un área importante para la conservación de las aves (AICA) en Colombia. Caldasia, 27(2), 247-265.

León-Lleras, J. y Rodríguez-Villamil, D. (2015). Registros del ibis pico de hoz, Plegadis falcinellus (Linnaeus 1776) (Pelecaniformes: Threskiornithidae) en Colombia. Biota Colombiana, 16(2), 158-161.

López, J. P. y Jiménez, M. (2009). Avifauna. En Reserva Forestal Protectora Bosque Oriental de Bogotá. Inventario de Fauna. Aves (47-51p). Bogotá D. C.: Conservación Internacional Colombia, Corporación Autónoma Regional de Cundinamarca.

López-O, J. P., Avendaño, J. E, Gutiérrez-Pinto, N. y Cuervo, A. M. (2014). The birds of the serranía de Perijá: The northernmost avifauna of the Andes. Ornitología Colombiana, 14, 62-93.

Lozano, I. (1993). Diversidad y organización en gremios de la comunidad de aves del sotobosque de bosque primario y vegetación secundaria. En Andrare, G. (Eds.). Carpanta Selva Nublada y Páramo. Pp 141-154. Bogotá D. C.: Fundación Natura Colombia.

Márquez, C., Bechard, M., Gast, F. y Vanegas, V. H. (2005). Aves rapaces diurnas de Colombia. Bogotá D.C.: Instituto de Investigación de Recursos Biológicos Alexander von Humboldt.

Martínez-Vilalta, A., Motis, A., y Kirwan, G. M. (2016). Whistling Heron (Syrigma sibilatrix). En del Hoyo, J., Elliott, Sargatal, A., J., Christie, D. A y de Juana, E. (Eds.). Handbook of the Birds of the World Alive. Barcelona: Lynx Edicions. Recuperado de http://www.hbw.com/ node/52672 Revisado el 17 de febrero 2016. 
Mayorga, J. y Melo, J. (2014). Aves de la vereda San Gil, municipio de Gutiérrez, Cundinamarca. Revista Científica, 19, 118-133.

McNish, T. (2007). Las Aves de los Llanos de la Orinoquia. Colombia: M\&B Ltda. 302 pp.

McNish, T. (2010). Las aves de los humedales de la Sabana de Bogotá y sus alrededores. Colombia: M\&B Ltda. 152 pp.

Ministerio de Ambiente y Desarrollo Sostenible. (2017). Listado de las especies silvestres amenazadas que se encuentran en el territorio nacional de Colombia. Colombia. Resolución 1912 de septiembre de 2017. 38 pp.

Morales, M. y Armentera, D. (2013). Estado de conservación de los bosques de niebla de niebla de los Andes colombianos, un análisis multiescalar. Boletín Científico Museo de Historia Natural, 17 (1), 64-72.

Morales M., Otero, J., Van der Hammen, T., Torres, A., Cadena, C., Pedraza, C., Rodríguez, N., Franco, C., Betancourth, J. C., Olaya, E., Posada, E. y Cárdenas, L. (2007). Atlas de páramos de Colombia. Bogotá D. C.: Instituto de Investigación de Recursos Biológicos Alexander von Humboldt. 208 pp.

Morales-Rozo, A. y Pachón, Y. (2014). Avifauna en diferentes hábitats de la cuenca del río Fúquene (Cundinamarca), Colombia. Biota Colombiana, 15 (Supl.1), 161-173.

Moreno, N. y Camargo P. (2008). Estado actual de la avifauna en la Reserva Biológica Encenillo (RBE) Guasca-Cundinamarca (Trabajo de grado). Bogotá D. C.: Universidad INCCA de Colombia, programa de Biología. 96 pp.

Moreno-Palacios, M. (2014). Chloropipo flavicapilla. En Renjifo, L. M., Gómez, M. F., VelásquezTibatá, J., Amaya-Villarreal, A. M., Kattan, G. H., Amaya-Espinel, J. D. y Burbano-Girón, J. (Eds.). Libro rojo de aves de Colombia, Volumen I: bosques húmedos de los Andes y la costa Pacífica. Pp 272-274. Bogotá D.C.: Editorial Pontificia Universidad Javeriana e Instituto Alexander von Humboldt.
Munves, J. (1975). Birds of a highland clearing in Cundinamarca, Colombia. The Auk, 92, 307-321.

Naranjo, L. G. (2002a). Anas georgica. En Renjifo, L. M., Franco-Maya, A. M., Amaya-Espinel, J. D., Kattan, G. H. y López-Lanús, B. (Eds.). Libro rojo de aves de Colombia. Pp 93-95. Serie libros rojos de especies amenazadas de Colombia. Bogotá D.C.: Instituto de Investigación de Recursos Biológicos Alexander von Humboldt y Ministerio del Medio Ambiente.

Naranjo, L. G. (2002b). Anas cyanoptera. En Renjifo, L. M., Franco-Maya, A. M., Amaya-Espinel, J. D., Kattan, G. H. y López-Lanús, B. (Eds.). Libro rojo de aves de Colombia. Pp 96-100. Serie libros rojos de especies amenazadas de Colombia. Bogotá D.C.: Instituto de Investigación de Recursos Biológicos Alexander von Humboldt y Ministerio del Medio Ambiente.

Naranjo, L. G., Amaya, J. D., Eusse-González, D. y Cifuentes-Sarmiento, Y. (Eds.). (2012). Guía de las especies migratorias de la biodiversidad en Colombia. Aves. Vol. 1. Bogotá D.C.: Ministerio de Ambiente y Desarrollo Sostenible/WWF Colombia. 708 pp.

Olivares, A. (1969). Aves de Cundinamarca. Bogotá D.C.: Universidad Nacional de Colombia. Facultad de Ciencias. Instituto de Ciencias Naturales. 425 pp.

Parra-Hernández R. M., Carantón-Ayala, D. A., Sanabria-Mejía, J. S., Barrera-Rodríguez, L. F., Sierra-Sierra, A. M., Moreno-Palacios, M. C., Yate- Molina, W. S., Figueroa-Martínez, W. E., Díaz-Jaramillo, C., Flórez-Delgado, V. T., Certuche-Cubillos, J. K., Loaiza-Hernández, H. N. y Florido-Cuellar, B. A. (2007). Aves del municipio de Ibagué-Tolima, Colombia. Biota Colombiana, 8(2), 199-220.

Parra-Hernández, R. M., Roa, A., Figueroa, W. E., Do Santos, D. M., Muñoz, A. y Castillo, L. (2012). Aves del Guavio-Cundinamarca, Colombia. The Field Museum-Rapid Color Guides, 357.

Pérez-Emán, J. L., Perdigón, J., Gutiérrez-Pinto, N., Cuervo, A. M., Céspedes, L. N., Witt, C. C., y Cadena, C. D. (2017). An extinct hummingbird 
species that never was: a cautionary tale about sampling issues in molecular phylogenetics. bioRxiv, 149898.

Phillips, R. (2012). Un nido activo de la escasa águila solitaria Harpyhaliaetus solitarius en Belize. Spizaetus, 13: 2-8.

Pulgarín-R., Bayly, P, N. J. y Ellery, T. (2015). New records of Chimney Swift (Chaetura pelagica) in Bogotá, Eastern Andes of Colombia. Boletín SAO, 24, 1-3.

Ramón, J. (1930). Geografía elemental de Cundinamarca. Bogotá D.C.: Procuraduría de los hermanos. Segunda edición. 68 pp.

Rangel-Ch., J. O., Lowy-C, P. D. y Aguilar-P, M. (1997). La distribución de los tipos de vegetación en las regiones naturales de Colombia. En Rangel-Ch., J. O., Lowy-C, P. D. y Aguilar-P, M. Colombia Diversidad Biótica II. Tipos de vegetación en Colombia. Pp 383-402. Bogotá D.C.: Instituto de Ciencias Naturales, Universidad Nacional de Colombia y Ideam.

Rasmussen, P. C. y Collar, N. ( 2016). Whitewhiskered Puffbird (Malacoptila panamensis). En del Hoyo, J., Elliott, A., Sargatal, J., Christie, D. A. y de Juana, E. (Eds.). Handbook of the Birds of the World Alive. Barcelona: Lynx Edicions. Recuperado de http://www.hbw.com/ node/55972 Revisado el 1 de marzo 2016.

Remsen, J. V., de Juana, Jr y E. (2016). Pale-legged Hornero (Furnarius leucopus). En del Hoyo, J., Elliott, A., Sargatal, J., Christie, D. A. y de Juana, E. (Eds.). Handbook of the Birds of the World Alive. Barcelona: Lynx Edicions. Recuperado de http://www.hbw.com/node/55972 Revisado el 1 de marzo 2016.

Renjifo, L. M., Franco-Maya, A. M., Amaya-Espinel, J. D., Kattan, G. H. y López-Lanús, B. (Eds.). (2002). Libro rojo de aves de Colombia. Serie libros rojos de especies amenazadas de Colombia. Bogotá D.C.: Instituto de Investigación de Recursos Biológicos Alexander von Humboldt y Ministerio del Medio Ambiente. 562 pp.

Renjifo, L. M., Gómez, M. F., Velásquez-Tibatá, J., Amaya-Villarreal, A. M., Kattan, G. H.,. Amaya-
Espinel, J. D.y Burbano-Girón, J. (2014). Libro rojo de aves de Colombia, Volumen I: bosques húmedos de los Andes y la costa Pacífica. Bogotá D.C.: Editorial Pontificia Universidad Javeriana e Instituto de Investigación de Recursos Biológicos Alexander von Humboldt. 465 pp.

Renjifo, L. M., Amaya-Villarreal, A. M., BurbanoGirón, J. y Velásquez-Tibatá, J. (2016). Libro rojo de aves de Colombia, Volumen II: Ecosistemas abiertos, secos, insulares, acuáticos continentales, marinos, tierras altas del Darién y Sierra Nevada de Santa Marta, y bosques húmedos del centro, norte y oriente del país. Bogotá D.C.: Editorial Pontificia Universidad Javeriana e Instituto de Investigación de Recursos Biológicos Alexander von Humboldt. 563 pp.

Ridgely, R. S. y Tudor, R. (2009). Field guide to the songbirds of South America. Austin, Texas The passerines. University of Texas Press, USA, $760 \mathrm{pp}$.

Rodríguez, D. Primer registro del Pato Cariblanco (Anas bahamensis) L. 1758 en los Andes de Colombia (En prensa). Boletín SAO.

Rodríguez-Calderón, J. A. (2011). Aves de las cascadas de Sueva y de la Reserva Natural Bosques Peña de Juiquín. Sueva Cundinamarca Colombia. Guía ilustrada. Bogotá D.C. 234 pp.

Rodríguez-Mahecha, J. V. (2002). Ara militaris. En Renjifo, L. M., Franco-Maya, A. M., AmayaEspinel, J. D., Kattan, G. y López-Lanús, B. (Eds.). Libro rojo de aves de Colombia. Serie libros rojos de especies amenazadas de Colombia. Pp 203206. Bogotá D.C.: Instituto de Investigación de Recursos Biológicos Alexander von Humboldt y Ministerio del Medio Ambiente.

Rojas, G. (2012). Dieta del guácharo Steatornis caripensis Humboldt, 1817 (Aves: Steatornithidae) en el Parque Nacional Natural Chingaza, CundinamarcaColombia (Trabajo de grado). Bogotá D.C.: Universidad Nacional de Colombia, posgrado en Ciencias-Biología. 81 pp.

Ruiz-Guerra, C. (2012a). Calidris fuscicollis. En Naranjo, L. G., Amaya, J. D., Eusse-González, D. y Cifuentes-Sarmiento, Y. (Eds.). Guía de las 
especies migratorias de la biodiversidad en Colombia. Aves. Vol. 1. Pp 233-235. Bogotá D.C.: Ministerio de Ambiente y Desarrollo Sostenible / WWF Colombia

Ruiz-Guerra, C. (2012b). Calidris bairdii. En Naranjo, L. G., Amaya, J. D., Eusse-González, D. y Cifuentes-Sarmiento, Y. (Eds.). Guía de las especies migratorias de la biodiversidad en Colombia. Aves. Vol. 1 Pp 236-238. Bogotá D.C.: Ministerio de Ambiente y Desarrollo Sostenible / WWF Colombia.

Ruiz-Guerra, C. y Estela, F. (2016). Anas bahamensis. En Renjifo, L. M.,. AmayaVillarreal, A. M, Burbano-Girón, J. y VelásquezTibatá, J. Libro rojo de aves de Colombia, Volumen II: Ecosistemas abiertos, secos, insulares, acuáticos continentales, marinos, tierras altas del Darién y Sierra Nevada de Santa Marta, y bosques húmedos del centro, norte y oriente del país. Pp 423-426. Bogotá D.C.: Editorial Pontificia Universidad Javeriana e Instituto Alexander von Humboldt.

Salaman, P. G. W., Stiles, F. G., Bohórquez, C. I., Álvarez-R, M., Umaña, A. M., Donegan, T. M., Cuervo, A. M. (2002). New and noteworthy bird records from the east slope of the Andes of Colombia. Caldasia, 24(1), 157-189.

Snow, D. y de Juana, E. (2017). Wing-barred Piprites (Piprites chloris). En del Hoyo, J., Elliott, A., Sargatal, J., Christie, D. A. y de Juana, E. (Eds.). Handbook of the Birds of the World Alive. Barcelona. Lynx Edicions. Recuperado de https://www.hbw.com/node/57114 Revisado el 7 de noviembre 2017.

Stiles, F. G. (1998). Avifauna. En Linares, E. (Ed.). Reconocimiento ecológico preliminar de la biota de la cuenca del Guavio (Informe técnico). Pp 257-295. Bogotá: Corporación Autónoma Regional del Guavio (Corpoguavio) - Instituto de Ciencias Naturales (Universidad Nacional de Colombia).

Stiles, F. G y Rosselli, L. (1998). Inventario de las aves de un bosque altoandino: Comparación de dos métodos. Caldasia, 20(1), 29-43.

Sua, A. y Chaparro-Herrera, S. (2010). Composición y estructura gremial del ensamblaje de aves de la vereda La Unión en el municipio de Quipile, departamento de Cundinamarca (Trabajo de grado) Bogotá D. C.: Universidad Pedagógica Nacional, departamento de Biología. 83 pp.

Turner, A. y Kirwan, G. M. (2016). White-thighed Swallow (Neochelidon tibialis). En del Hoyo, J., A. Elliott, Sargatal, J., Christie, D. A. y de Juana, E. (Eds.). Handbook of the Birds of the World Alive. Barcelona: Lynx Edicions. Recuperado de http:/ / www.hbw.com/node/57725 Revisado el 18 de febrero 2016.

Vásquez, V. H. y Serrano, M. (2009). Las áreas naturales protegidas de Colombia. Bogotá D.C.: Conservación Internacional-Colombia y Fundación Biocolombia. 696 pp.

Zamudio, J. (2012). Leucophaeus pipixcan. En Naranjo, L. G., Amaya, J. D., Eusse-González, D. y Cifuentes-Sarmiento, Y. (Eds.). Guía de las especies migratorias de la biodiversidad en Colombia. Aves. Vol. 1. Pp. 262-264. Bogotá D.C.: Ministerio de Ambiente y Desarrollo Sostenible/ WWF Colombia.

Zimmer, K. y Isler, M. L. (2016a). White-shouldered Antshrike (Thamnophilus aethiops). En del Hoyo, J., Elliott, A., Sargatal, J., Christie, D. A. y de Juana, E. (Eds.). Handbook of the Birds of the World Alive. Barcelona: Lynx Edicions. Recuperado de http://www.hbw.com/node/56674 Revisado el 7 de marzo 2016.

Zuluaga-Bonilla, J. E. y Macana-García D. C. (2016). La avifauna actual del lago de Tota, Boyacá, Colombia: área importante para la conservación de las aves. Biota Colombiana, 17(2), 138-162.

Anexos 1-6. Disponible en línea: http:/ / revistas.humboldt.org.co/index.php/biota/rt/suppFiles/576/0 


\section{Sergio Chaparro-Herrera}

(Autor de correspondencia)

Asociación Bogotana de Ornitología (ABO)

Universidad de Antioquia,

Grupo de Ecología y Evolución de Vertebrados Medellín, Colombia

sergioupn@gmail.com

\section{Andrea Lopera-Salazar}

Universidad de Antioquia,

Grupo de Ecología y Evolución de Vertebrados

Medellín, Colombia

alopera4@gmail.com

\section{F. Gary Stiles}

Universidad Nacional de Colombia,

Instituto de Ciencias Naturales

Bogotá, Colombia

fgstilesh@unal.edu.co
Aves del departamento de Cundinamarca, Colombia: conocimiento, nuevos registros y vacíos de información

Citación del artículo: Chaparro-Herrera, S., Lopera-Salazar, A. y Stiles, F. G. (2018). Aves del departamento de Cundinamarca, Colombia: conocimiento, nuevos registros y vacíos de información. Biota Colombiana, 19(1), 160-189. DOI: 10.21068/c2018.v19n01a11.

Recibido: 24 de agosto de 2017

Aprobado: 4 de abril de 2018 